Advanced Functional Materials, 24(26):4068-4081, 2014

DOI: 10.1002/adfm.201304216 (†)

Article type: Full Paper

\title{
Correlated Donor/Acceptor Crystal Orientation Controls Photocurrent Generation in All-Polymer Solar Cells
}

Marcel Schubert, Brian A. Collins, Hannah Mangold, Ian A. Howard, Wolfram Schindler, Koen Vandewal, Steffen Roland, Jan Behrends, Felix Kraffert, Robert Steyrleuthner, Zhihua Chen, Konstantinos Fostiropoulos, Robert Bittl, Alberto Salleo, Antonio Facchetti, Frédéric Laquai, Harald W. Ade, and Dieter Neher*

Marcel Schubert, Steffen Roland, Robert Steyrleuthner, and Prof. Dr. Dieter Neher University of Potsdam, Institute of Physic and Astronomy, 14476 Potsdam, Germany neher@uni-potsdam.de

Dr. Brian A. Collins, and Prof. Dr. Harald W. Ade

North Carolina State University, Department of Physics, Raleigh, NC 27695, USA

Dr. Brian A. Collins

National Institute of Standards and Technology (NIST), Gaithersburg, USA

Hannah Mangold, Dr. Ian A. Howard, and Dr. Frédéric Laquai

Max-Planck-Institute for Polymer Research (MPI-P), 55128 Mainz, Germany

Dr. Koen Vandewal, and Prof. Dr. Alberto Salleo

Stanford University, Department of Materials Science, Stanford, CA 94305-4034, USA

Dr. Wolfram Schindler and Dr. Konstantinos Fostiropoulos

Helmholtz-Zentrum Berlin (HZB), Institute for Heterogeneous Material Systems, 14109

Berlin, Germany

Dr. Jan Behrends, Felix Kraffert, Robert Steyrleuthner, and Prof. Dr. Robert Bittl

Free University Berlin, Department of Physics, 14195 Berlin, Germany

Dr. Zhihua Chen and Prof. Dr. Antonio Facchetti

Polyera Corporation, Illinois 60077, USA

Keywords: photocurrent generation, charge-transfer state recombination, polymer crystals, alternative acceptors, organic solar cells

$(\dagger)$ Disclaimer: the publisher's version overrides in case of any discrepancy: 


\section{Abstract}

New polymers with high electron mobilities have spurred research in organic solar cells using polymeric rather than fullerene acceptors due to their potential of increased diversity, stability and scalability. However, all-polymer solar cells have struggled to keep up with the steadily increasing power conversion efficiency of polymer:fullerene cells. The lack of knowledge about the dominant recombination process as well as the missing concluding picture on the role of the semi-crystalline microstructure of conjugated polymers in the free charge carrier generation process impede a systematic optimization of all-polymer solar cells. We examine these issues by combining structural and photo-physical characterization on a series of poly(3hexylthiophene) (donor) and P(NDI2OD-T2) (acceptor) blend devices. Our experiments reveal that geminate recombination is the major loss channel for photo-excited charge carriers. Advanced X-ray and electron-based studies reveal the effect of chloronaphthalene co-solvent in reducing domain size, altering domain purity and reorienting the acceptor polymer crystals to be coincident with those of the donor. This reorientation correlates well with the increased photocurrent from these devices. Thus, efficient split-up of geminate pairs at polymer/polymer interfaces may necessitate correlated donor/acceptor crystal orientation, which represents an additional requirement compared to the isotropic fullerene acceptors. 


\section{Introduction}

Conjugated polymers represent the most versatile material class in the field of organic photovoltaics. Their great chemical variability allows the modification of their optical, mechanical, energetic and charge transporting properties. Polymers with large electron affinities are being designed in an attempt to rival fullerene as the electron accepting moiety in organic solar cells (OSCs). Over the last decade, fullerenes have been used in almost all efficiency-breaking, solution-processed OSCs, ${ }^{[1-3]}$ while devices incorporating polymeric acceptors lag significantly behind their fullerene counterparts, with modest power conversion efficiencies (PCE) between 3 and $4 \%$ at best. ${ }^{[4-8]}$ A low external quantum efficiency (EQE) is primarily responsible for the poor performance of such all-polymer devices. To the best of our knowledge, the highest reported EQE of an all-polymer solar cell does not exceed $50 \%{ }^{[8]}$ In general, the primary excitation formed by photon absorption in an organic solar cell is a strongly bound, Frenkel-type singlet exciton. Charge transfer at a donor/acceptor heterojunction is so far the most efficient process to overcome the Coulombic interactions of this excited electron-hole pair, and produce spatially separated charges. However, charge transfer does not necessarily lead to free, uncorrelated electrons and holes. Rather, Coulombically bound interfacial electronic states can be formed that strongly influence the energy conversion efficiency of the OSC. The term charge-transfer state (CTS) is commonly used to describe such weakly-interacting interfacial excited-state species. ${ }^{[9]}$ Often a CTS has weak optical features discernable in the luminescence or absorbance spectra of D/A mixtures which assists in photophysical characterization of their properties and influence on device physics. For example this spectral information was used to disclose a direct correlation of CTS energy and open circuit voltage $\left(V_{\mathrm{OC}}\right) .{ }^{[10]}$ In blends of conjugated polymers, previous work has assigned geminate recombination via CTS as a dominant pathway for the decay of the photo-induced excitations. ${ }^{[11-14]}$ Thus, the low EQE, generally observed in all-polymer solar cells, suggests that geminate recombination is a common process in polymer-polymer blends.

It has been recently suggested that the efficiency of photoexcited exciton to free electron conversion is controlled by the extent of charge delocalization on the donor or acceptor. ${ }^{\text {[15-19] }}$ However, the specific structural or electronic properties that assist exciton dissociation remain unclear. For many years, the high electron mobility was an almost exclusive feature of fullerenes in OSCs, ${ }^{[20,21]}$ especially when compared to common acceptor polymers, ${ }^{[6,12,22]}$ and a possible key to their superior performance. ${ }^{[18,23]}$ In the last years several conjugated 
polymers with excellent electron mobility have been discovered. ${ }^{[24-29]}$ The prototype is the donor/acceptor copolymer P(NDI2OD-T2) which reaches mobilities of $0.85 \mathrm{~cm}^{2} \mathrm{~V}^{-1} \mathrm{~s}^{-1}$ and $5 \times 10^{-2} \mathrm{~cm}^{2} \mathrm{~V}^{-1} \mathrm{~s}^{-1}$, in organic field-effect transistors (OFET) and single carrier diodes, respectively. ${ }^{[24,30,31]}$ In addition, the large electron affinity of about $-4 \mathrm{eV}$, the low optical bandgap of $1.45 \mathrm{eV}$ and the manifold of available data on charge transport ${ }^{[24,30-32]}$, microscopic structure ${ }^{[32-34]}$ and optoelectronic properties ${ }^{[35]}$ render P(NDI2OD-T2) a good candidate as model electron-accepting material. Surprisingly, despite the excellent properties of the neat P(NDI2OD-T2) polymer, the first results of photoactive bilayers and blends of $\mathrm{P}(\mathrm{NDI} 2 \mathrm{OD}-\mathrm{T} 2)$ in combination with P3HT as the donor were surprisingly poor, with PCEs ranging between 0.02 and $0.6 \% .{ }^{[36-39]}$ By incorporating chloronaphthalene $(\mathrm{CN})$ as co-solvent and applying a high temperature drying process during film formation, we could increase the efficiency to $1.4 \%{ }^{[40]}$ Despite the higher efficiency and excellent light harvesting properties, the solar cells were still limited by the modest EQE of about $20 \%$.

It is the aim of this paper to address the reasons for the low photocurrent generation yields of blends of the donor P3HT and the acceptor P(NDI2OD-T2). Special emphasis is paid to the processes controlling free charge carrier generation to guide future improvements of allpolymer solar cells. Through variation of processing parameters, the magnitude of photocurrent produced by the solar cells was systematically explored and correlated to changes of the orientation of polymer crystallites by advanced structural and photo-physical characterization. Our analysis suggests that face-to-face staking of the D/A polymer crystals is necessary to generated free charges, while misoriented chains inescapably cause recombination of geminate charge carrier pairs. Furthermore, we observe that exciton selftrapping in the crystalline acceptor phase represents an additional major loss channel. Our results imply that an optimized relative orientation of the D/A crystallites is required to achieve high quantum efficiencies in all-polymer solar cells.

\section{Results and Discussion}

\subsection{Solar cell performance}

Firstly, the impact of film preparation conditions on the solar cell performance will be presented. We have previously shown that the mixture of solvents from which the active layer is cast has a large influence on the device performance. Specifically, we found that the amount of chloronaphthalene $(\mathrm{CN})$ in the solution mixture with xylene (Xy) was a critical parameter in the solar cell optimization process. ${ }^{[40]}$ Figure 1.a shows solar cell characteristics for different mixing ratios of $\mathrm{Xy}$ and $\mathrm{CN}$. Both $J_{\mathrm{SC}}$ and $P C E$ are found to increases with 
increasing $\mathrm{CN}$ content by about one order of magnitude and reach a maximum of $3 \mathrm{~mA} / \mathrm{cm}^{2}$ and $1.1 \%$, respectively, at 50 vol. $\% \mathrm{CN}$. In a recent study we could demonstrate that the high boiling point of the solvent $\mathrm{CN}$ suppresses the pre-aggregation of $\mathrm{P}(\mathrm{NDI} 2 \mathrm{OD}-\mathrm{T} 2)$ in solution. ${ }^{[35,40]}$ On the other hand, we see only slight changes in solar cell performance when the co-solvent Xy is replaced by other solvents (Figure S1). Thus, the increase in photocurrent is mainly due to the addition of $\mathrm{CN}$, while the co-solvent is only needed to prevent dewetting ${ }^{[40]}$ of the film during the drying step at $200^{\circ} \mathrm{C}$. After drying, the performance is not affected by further thermal annealing. Even a $1 \mathrm{~h}$ solvent annealing step at elevated temperatures leaves the performance of the best blends unchanged (Figure S2), which demonstrates the excellent thermal stability of the optimized P3HT:P(NDI2OD-T2) blends. A remarkable feature of the devices presented in Figure 1.a is the consistently high fill factor, ranging from $62 \%$ to $65 \%$ despite a $300 \mathrm{~nm}$ film thickness. This indicates that the bias voltage dependence of the photocurrent is small, which has important implications for our understanding of the device physics. Namely, it indicates that the electric field and voltage dependence of free charge carrier generation and recombination are almost negligible up to the voltage which produces the maximum output power. We will investigate this in more details in a forthcoming study. In the further experimentation and analysis, we will elucidate on the limitations of the short-circuit currents, where the external $(E Q E)$ and internal quantum efficiency $(I Q E)$ measurements will give a first insight.
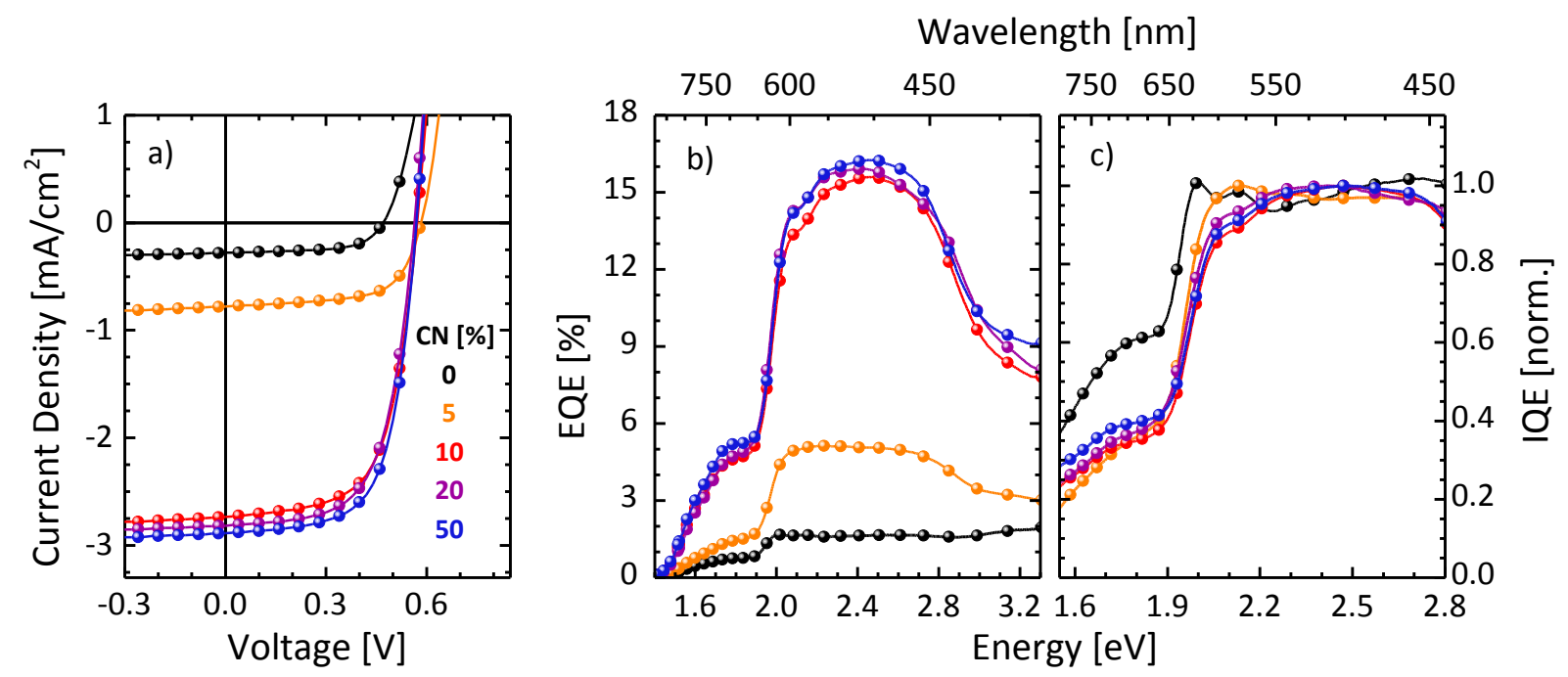

Figure 1 a) Current density-voltage characteristics of P3HT:P(NDI2OD-T2) solar cells prepared from $\mathrm{Xy}: \mathrm{CN}$ solvent mixtures, measured under AM $1.5 \mathrm{G}$ conditions. The $\mathrm{CN}$ concentration is indicated in the graph. The active layer thickness and donor:acceptor composition was held constant at $300 \mathrm{~nm}$ and 1:0.75, respectively. b) EQE and c) normalized IQE spectra of the same devices.

The EQE spectra are given in Figure 1.b. The maximum EQE varies between the cells proportional to their photocurrent and reaches $16 \%$ in the best performing cell. All spectra 
have a similar shape and composition. The maximum EQE is measured between 2.8 and $2.0 \mathrm{eV}$, i.e. the range where $\mathrm{P} 3 \mathrm{HT}$ constitutes the maximum absorption. In the range of maximum P(NDI2OD-T2) absorbance and the absence of P3HT absorption, from $1.9 \mathrm{eV}$ to $1.6 \mathrm{eV}$, the EQE drops significantly to about one third of the maximum value. However, in order to compare the photon-to-electron conversion yields more meaningfully, we calculated the internal quantum efficiency (IQE), which is the EQE corrected by the fraction of photons absorbed in the active layer. In absolute values, the maximum EQE and IQE are almost identical and differ by less than one percent in the range displayed in Figure 1.c. This is a consequence of the large film thickness. Here, we make a first conclusion that the low IQE of the P3HT:P(NDI2OD-T2) solar cells is responsible for the low photocurrent and represents the device limiting processes. To compare the spectral shape of the IQE, Figure 1.c shows the normalized spectra of all devices. The IQE in the P(NDI2OD-T2) phase is only between $40 \%$ (in the $\mathrm{CN}$-cast devices) and $60 \%$ (in the $\mathrm{Xy}$-cast device) of the value measured in the P3HT domains. Thus, beside the overall small maximum IQE values, an additional process further lowers the charge carrier generation efficiency of excitons absorbed by P(NDI2OD-T2) as compared to excitations absorbed by P3HT.

Table 1 Averaged solar cell parameters (numbers in parentheses denote the uncertainty in the last digit), photoluminescence quenching efficiency (PQE) and active layer thickness $d$ (numbers in square brackets denote the root-mean square roughness measured by atomic force microscopy) of the different P3HT:P(NDI2OD-T2) blend films.

\begin{tabular}{|c|c|c|c|c|c|c|c|}
\hline $\mathrm{Xy}: \mathrm{CN}$ & Drying & $\mathrm{Jsc}_{\mathrm{sc}}\left[\mathrm{mA} / \mathrm{cm}^{2}\right]$ & $F F[\%]$ & $V_{O C}$ & $\eta[\%]$ & $\begin{array}{c}\text { PQE (\%) } \\
\text { (P3HT/ } \\
\text { P(NDI2OD-T2)) }\end{array}$ & $\begin{array}{c}d[\mathrm{RMS}] \\
{[\mathrm{nm}]}\end{array}$ \\
\hline 100:0 & as cast & $0.27(1)$ & $62(1)$ & $0.47(1)$ & $0.08(1)$ & $91.9 / 44.7$ & 300 [15] \\
\hline $95: 5$ & dry@200C & $0.77(6)$ & $64(1)$ & $0.58(1)$ & $0.29(2)$ & 91.5 / - & 300 [27] \\
\hline $90: 10$ & " & $2.8(1)$ & $63(1)$ & $0.57(1)$ & $0.99(3)$ & $94.9 /-$ & $295[6]$ \\
\hline $80: 20$ & $"$ & $2.9(2)$ & $62(1)$ & $0.56(1)$ & $1.00(6)$ & $95.6 /-$ & 300 [6] \\
\hline 50:50 & " & $2.9(2)$ & 65 (1) & $0.57(1)$ & $1.07(8)$ & 96.2 / 54.8 & $300[4]$ \\
\hline
\end{tabular}

\subsection{Charge-transfer states}

In order to address the processes limiting the IQE of our devices, EQE measurements were combined with photothermal deflection spectroscopy (PDS) over a wide spectral range, including the absorption by charge-transfer states. Recent work by Vandewal et al. suggested that free carrier generation proceeds via the fully-relaxed CTS. ${ }^{[41]}$ Figure 2 displays the logarithmically plotted EQE, $A$ and $I Q E$ spectra of a 50 vol.\% $\mathrm{CN}$-cast blend, measured from 
the visible absorption of P3HT, then P(NDI2OD-T2), down to the near infrared (NIR) where the signal is governed by direct excitation of the interfacial charge-transfer states. ${ }^{[42]}$ In the EQE spectrum, the two characteristic regions of P3HT and P(NDI2OD-T2), as they are shown in Figure 1.b, can be easily identified. Below the bandgap of P(NDI2OD-T2) a pronounced shoulder appears which originates from the excitation of the CTS. ${ }^{[10,43-45]}$ The weak CTS absorption could be detected down to energies of about $1.2 \mathrm{eV}$, before it disappears behind the background signal of the PDS setup.

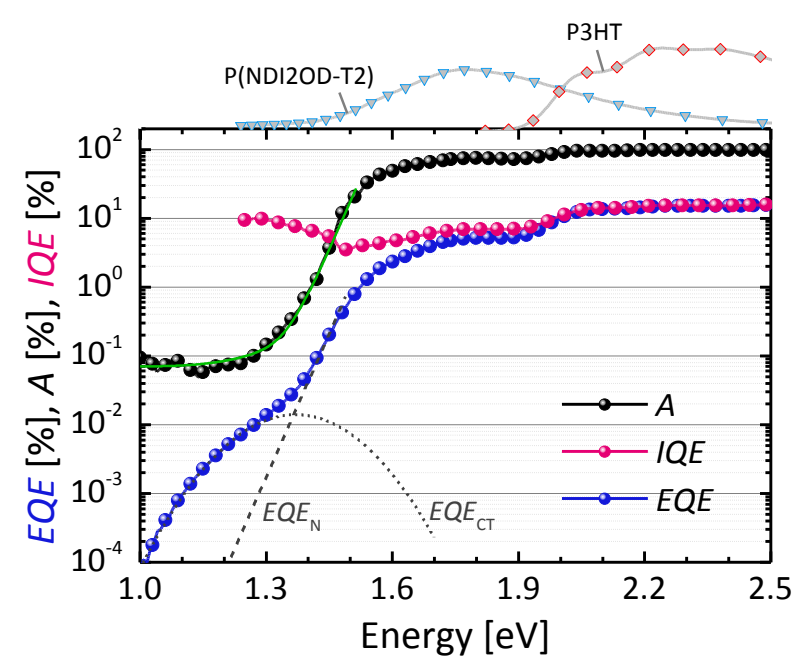

Figure 2 EQE (blue spheres), IQE (pink spheres) and active layer absorption $A$ (black spheres) of the 50:50 Xy:CN-cast blend. On top of the figure, the absorbance spectra of pure $\mathrm{P} 3 \mathrm{HT}$ (red diamonds) and $\mathrm{P}$ (NDI2OD-T2) (blue triangles) are given. The main contribution to the IQE in the range up to $1.5 \mathrm{eV}$, from 1.5 to $1.9 \mathrm{eV}$ and above $1.9 \mathrm{eV}$ are assigned to CTS, P(NDI2OD-T2) and P3HT, respectively. Also shown are fits to the CTS region ( $E Q E_{C T}$, gray dotted line) and the P(NDI2OD-T2) band gap region $\left(E Q E_{N}\right.$, gray dashed line), as well as the best fit to the absorption (green line), assuming an $I Q E_{\mathrm{CT}}$ of $35 \%$.

To estimate the IQE upon direct CTS excitation, we separated the EQE into the contribution originating from the CTS and from the P(NDI2OD-T2). We then recalculated the absorbance $A_{\mathrm{NIR}}$ in the NIR region from the modelled EQE spectrum, using the IQE as a variable fit parameter and taking into account the background signal (for details see Supporting Information). The best fit is obtained when the IQE of the CTS $\left(I Q E_{\mathrm{CT}}\right)$ is equal to $35 \%$ (see green line in Figure 2), although it is not possible to define an upper boundary. This important result suggests that the CTS can split with significantly higher efficiency than the direct excitation of either the donor or acceptor polymer. It shows that the CTS generates free charge carriers, as it has been demonstrated very recently for several D/A combinations. ${ }^{[41]}$ However, whereas the IQE of a directly-excited CTS in previous studies was equal to the internal quantum efficiency based on absorption by the donor or acceptor, ${ }^{[41,42]}$ we find a larger value for the P3HT:P(NDI2OD-T2) blend investigated here. 


\subsection{Blend domain structure}

To elucidate the influence of $\mathrm{CN}$ on the all-polymer blend film morphology, we combined resonant soft X-ray scattering (R-SoXS) and energy-filtered transmission electron microscopy (EF-TEM) measurements. Compared to conventional TEM, where the contrast relies mainly on the difference in mass density, EF-TEM shows enhanced chemical contrast. We analyzed the electron energy loss spectrum at the sulphur ionization edge $(165 \mathrm{eV})$, where the interaction of the transmitted electrons with the sulphur containing thiophene units reduces their kinetic energy. Due to the three times larger sulphur density of P3HT compared to $\mathrm{P}(\mathrm{NDI} 2 \mathrm{OD}-\mathrm{T} 2)$, energy filtering provides sufficient chemical contrast between these polymers. Consequently, bright areas correspond to P3HT-rich domains, while dark areas are $\mathrm{P}(\mathrm{NDI} 2 \mathrm{OD}-\mathrm{T} 2)$-rich domains. The EF-TEM sulphur map of the Xy-cast blend film is given in Figure 3.a. It displays a strongly phase separated morphology comprised of irregular shaped P3HT domains with a typical diameter of $1 \mu \mathrm{m}$, and a network of P(NDI2OD-T2) fibers, which have a width of $0.5-1 \mu \mathrm{m}$. Additional scanning transmission X-ray microscopy (STXM) measurements reveal that the darkest regions in EF-TEM sulphur maps are regions of pure $\mathrm{P}(\mathrm{NDI} 2 \mathrm{OD}-\mathrm{T} 2)$ which probably represent crossing points of several fiber bundles (see Figure S4). From the EF-TEM, STXM and additional scanning Kelvin probe microscopy (SKPM, see Figure S5) data it is also apparent that the P(NDI2OD-T2) fiber bundles are embedded within a P3HT matrix. Furthermore, the P3HT domains that do not overlap with $\mathrm{P}(\mathrm{NDI} 2 \mathrm{OD}-\mathrm{T} 2)$ fibers, visible as the brightest domains in the EF-TEM elemental maps, show an average P3HT concentration between 80 and $90 \mathrm{wt} \%$ (see line scans of STXM composition maps in Figure S4), indicating that these domains are rather impure and contain a significant fraction of $\mathrm{P}(\mathrm{NDI} 2 \mathrm{OD}-\mathrm{T} 2)$. The domain structure of the Xy-cast film is consistent with previous studies that used either STXM or near-field scanning optical microscopy (NSOM). ${ }^{[38,40]}$ However, the resolution of the NSOM and STXM was too small to disclose the morphology of the $\mathrm{Xy}: \mathrm{CN}$-cast films. A sufficient resolution is provided by EF-TEM and Figure 3.b reveals that the addition of $\mathrm{CN}$ strongly affects the structure of the P(NDI2OD-T2) domains. Compared to the micrometer large fiber network in the Xy-cast blend, P(NDI2ODT2) arranges in circular domains in the 50:50 Xy:CN-cast film. Embedded in a P3HT matrix, these domains have a typical diameter between 30 and $50 \mathrm{~nm}$, rather independent on the active layer thickness (see EF-TEM thickness series in Figure S6). 
a)
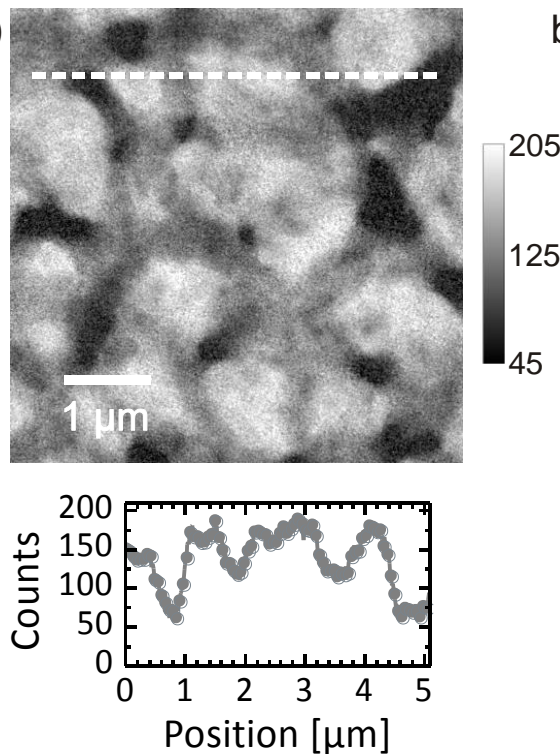

b)
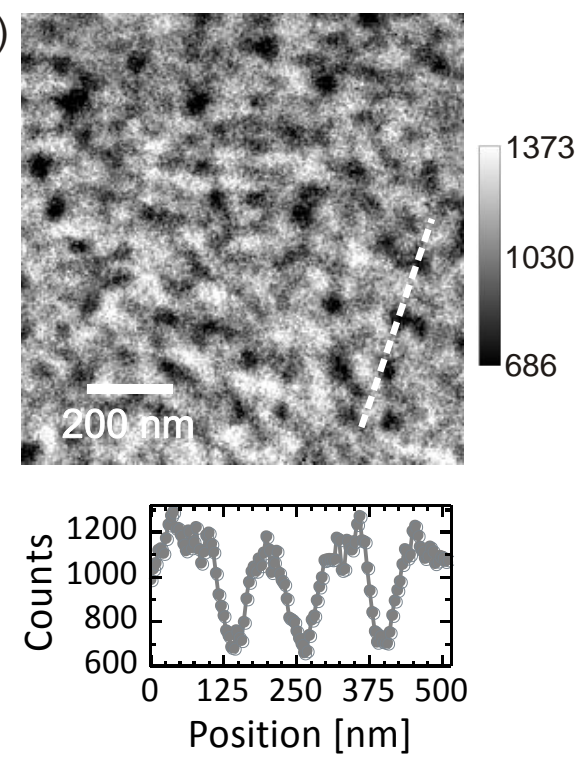

Figure 3 EF-TEM sulphur maps of $\boldsymbol{a}$ ) Xy-cast and $\boldsymbol{b})$ 50:50 Xy:CN-cast P3HT:P(NDI2OD-T2) blends. The signal is expressed in sulphur counts, where bright and dark areas correspond to P3HT- and $\mathrm{P}(\mathrm{NDI}$ 2OD-T2)-rich domains, respectively. The thickness of the film in $a$ ) and b) is $170 \mathrm{~nm}$ and $145 \mathrm{~nm}$, respectively. Dashed lines indicate line scans shown below the images.

To investigate more quantitatively and with a higher resolution the morphology transition within the whole blend series resulting from varying the $\mathrm{CN}$ concentrations, R-SoXS experiments were performed. Figure 4.a shows profiles acquired at an energy of $285.3 \mathrm{eV}$, where the maximum chemical contrast between both polymers is observed. ${ }^{[46,47]}$ Profiles acquired at $270 \mathrm{eV}$ are also shown. These correspond to the non-resonant conditions in conventional small angle X-ray scattering (SAXS) and are more sensitive to mass density fluctuations. For the Xy-cast film the R-SoXS profile shows the highest intensity at $q=0.0045 \mathrm{~nm}^{-1}$ and a shoulder at $q=0.014 \mathrm{~nm}^{-1}$. The corresponding characteristic lengths $l_{\mathrm{c}}=2 \pi / q$ of 1.4 and $0.44 \mu \mathrm{m}$ can be retraced in the EF-TEM maps as the domain spacing and the mesh width of the P(NDI2OD-T2) network, respectively. However, both features exhibit rather broad distributions. In stark contrast to the Xy-cast film, the R-SoXS profile of the 50 vol.\% CN-cast sample consists of a broad feature at $q \approx 0.08-0.09 \mathrm{~nm}^{-1}\left(l_{\mathrm{C}}=70 \mathrm{~nm}\right)$ and a shoulder at $q \approx 0.2 \mathrm{~nm}^{-1}\left(l_{\mathrm{c}}=35 \mathrm{~nm}\right)$, which we attribute to particle-like scattering from the circularly shaped domains seen in the EF-TEM sulphur maps. Furthermore, an additional shoulder is identified in the pattern at lower values of $q$ where $\log$ normal peak fits to the profile yields a position of $q=0.056 \mathrm{~nm}^{-1}\left(l_{\mathrm{C}}=112 \mathrm{~nm}\right)$, reflecting the typical spacing of the $\mathrm{P}(\mathrm{NDI} 2 \mathrm{OD}-\mathrm{T} 2)$ domains seen in EF-TEM. The two high- $q$ features, caused by scattering from the smaller circularly shaped P(NDI2OD-T2) domains, are common in all films with any amount of $\mathrm{CN}$, linking the formation of these domains to the presence of the $\mathrm{CN}$. In contrast, the position of the low-q feature, interpreted as dominant domain spacing, changes with $\mathrm{CN}$ - 
concentration. Starting with $l_{C}=1.4 \mu \mathrm{m}$ at $0 \mathrm{vol} \%$, the feature moves to $l_{C}=690 \mathrm{~nm}$ with 5 vol. $\%$, and $l_{\mathrm{C}}=123 \mathrm{~nm}$ with $10 \mathrm{vol} . \%$. At higher $\mathrm{CN}$ concentration, the change in position stops, with the dominant domain spacing for $20 \mathrm{vol} \%$ and 50 vol.\% equal to each other at $l_{\mathrm{C}}=112 \mathrm{~nm}$. Thus from the scattering profiles, it is likely that the mesoscale structural changes in the film largely saturate between 10 and 20 vol.\% CN. STXM and SKPM on the film cast from 5 vol.\% CN (see Figure S3 and S4) resolves a structure with large P(NDI2OD-T2) domains reminiscent of the fibers seen in the film cast from pure $\mathrm{Xy}$. Thus, the 5 vol.\% CNcast film represents a transition morphology with coexisting remnant P(NDI2OD-T2) fibres and the emerging population of smaller circularly shaped domains.
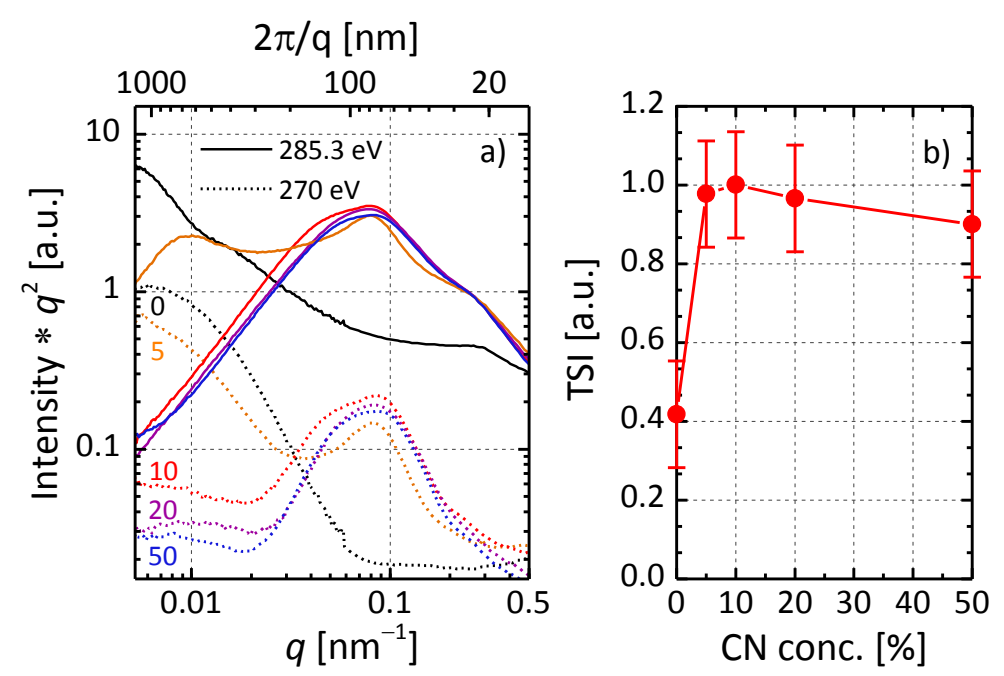

Figure 4 a) R-SoXS and SAXS profiles of blend layers with $300 \mathrm{~nm}$ thickness, measured at the $\mathrm{C}_{1 \mathrm{~s}}-\pi^{*}$ transition energy ( $285.3 \mathrm{eV}$, solid lines) and at non-resonant energy ( $270 \mathrm{eV}$, dashed lines). The CN concentration of the $\mathrm{Xy}: \mathrm{CN}$ casting solution is indicated in the graph. $\boldsymbol{b})$ Calculated total scattering intensity (TSI) from integrating the R-SoXS profiles shown in a) with uncertainties calculated from that of the film thickness and power law extensions to higher $\mathrm{q}$.

In Figure 4.b. the total scattering intensity (TSI) has been calculated for each profile after extension to higher q with power-law fits, allowing for convergence of the integrals. As a function of $\mathrm{CN}$-concentration, the TSI increases strongly upon addition of 5 vol.\% $\mathrm{CN}$ and then remains the same for higher $\mathrm{CN}$ concentrations. This indicates that the $\mathrm{CN}$-induced domains have a higher purity than those cast from pure xylene. Combined with the fact that the STXM analysis in Xy-cast films showed pure fibers of P(NDI2OD-T2) but impure P3HT domains, the rise in TSI suggests that mainly the purity of the P3HT domains increases upon addition of $\mathrm{CN}$. Consequently, the smaller P(NDI2OD-T2) domains in the CN-cast blends are similarly pure. This conclusion is supported by the large contrast in the sulphur maps, which can be seen more clearly in the line scans in Figure 3. 


\subsection{Polymer crystallinity}

It has been presumed that the generation of free charge carriers is dictated by the microscopic structure of the donor and acceptor at the heterojunction. ${ }^{[19,48,49]}$ P3HT and P(NDI2OD-T2) are semi-crystalline materials, ${ }^{[33,50]}$ which comprise well-ordered aggregates or crystallites, as well as disordered amorphous fractions. While ordered regions are associated with better charge transport and larger exciton and charge delocalization, it is generally unknown which role they play in the exciton dissociation process.

Grazing-incidence wide-angle X-ray scattering (GIWAXS) measurements have been used to study the microstructure of the crystalline domains. The different molecular structure of both polymers gives rise to a relatively large number of well separated scattering peaks, which allows the extraction of the molecular packing structure, the size and orientation of the P3HT and P(NDI2OD-T2) crystallites. Figure 5 displays 2D plots as well as sector averaged GIWAXS profiles of the $\mathrm{Xy}$ - and $\mathrm{Xy}: \mathrm{CN}$-cast blends. According to the usual convention, the crystal directions were labeled as lamella stacking [h00], $\pi-\pi$ stacking [0k0] and backbone repeat [00l], while the letter P and $\mathrm{N}$ of the peak labels stands for P3HT and P(NDI2OD-T2), respectively. From the peak positions the real space repeat distances are obtained, which are in agreement with published values, ${ }^{[33]}$ while the coherence of P3HT and P(NDI2OD-T2) crystallites is obtained from a Scherer analysis of the first order peak width. Furthermore, we use the intense [100] lamella stacking peak to probe the orientation of the crystals relative to the substrate.
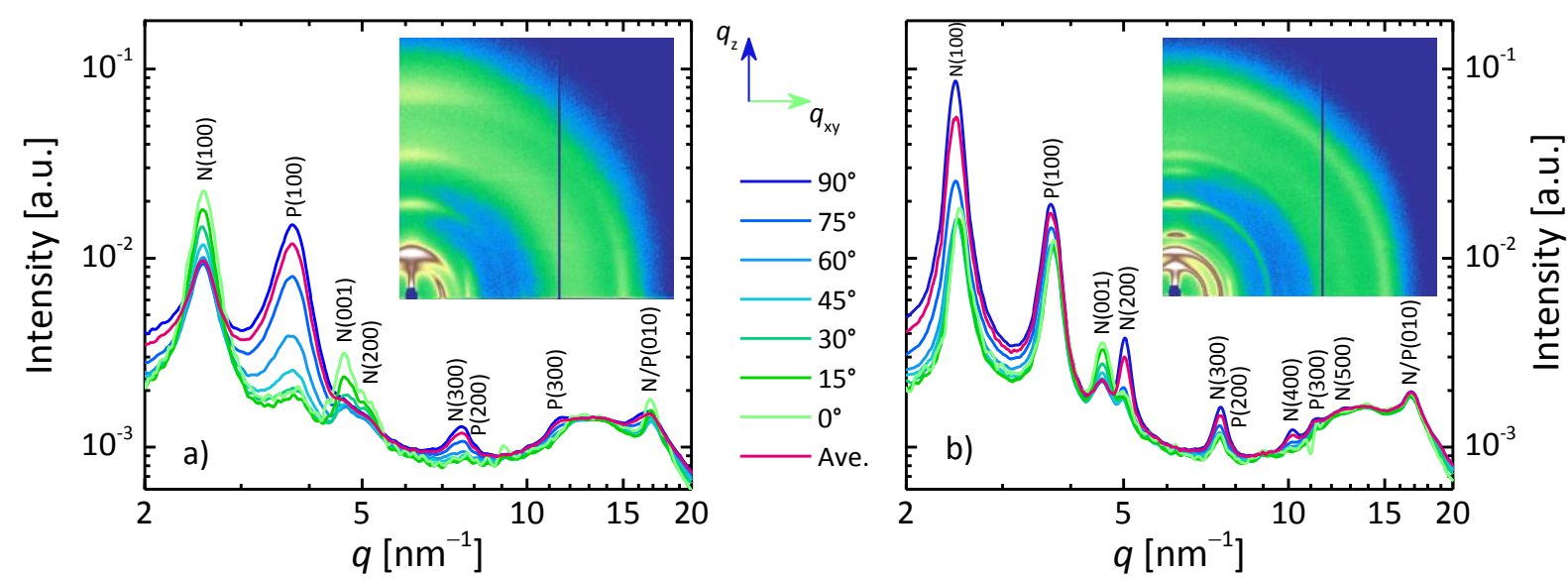

Figure 5 Sector averaged GIWAXS scattering intensity of the Xy- (left) and the 50 vol.\% CN-cast (right) blend. The center angles of the $15^{\circ}$ sectors are denoted between the graphs and range from in-plane scattering at $0^{\circ}\left(q_{\mathrm{xy}}\right.$, light green line) to out-of-plane scattering at $90^{\circ}\left(q_{z}\right.$, dark blue line). The 2D detector images are shown as insets. Peaks belonging to P3HT and P(NDI2OD-T2) are labeled with $\mathrm{P}$ and $\mathrm{N}$, respectively. 
We start with a quantitative description of the orientation distribution of the polymer crystals. From the two-dimensional GIWAXS data, pole figures were constructed following previously published procedures. A pole figure displays the distribution of crystals as a function of the polar angle $\omega$, defined as the angle between the substrate's surface normal and the [100] lamella stacking direction. ${ }^{[51,52]}$ Figure 6 shows the lamella stacking orientation distribution of P3HT and P(NDI2OD-T2). In the Xy-cast blend, P3HT crystals adopt a preferential edgeon orientation where the majority of crystals are oriented within a polar angle of $\pm 25^{\circ}$ degrees, while P(NDI2OD-T2) crystals are oriented diametrical opposed, adopting a face-on orientation with the P(NDI2OD-T2) backbone lying parallel to the substrate. The use of CN introduces two distinct changes to these distributions. The first is the reorientation of the $\mathrm{P}(\mathrm{NDI} 2 \mathrm{OD}-\mathrm{T} 2)$ crystals from face- to edge-on. This transition is clearly visible in the blend processed from 5 vol.\% CN. Here, a large face-on population of P(NDI2OD-T2) with a maximum at about $\omega=75^{\circ}$ coexists with an edge-on population. The change of the orientation distribution saturates at about $20 \mathrm{vol} \% \mathrm{CN}$, leaving a dominant edge-on population and a population with a random distribution, which is reflected by the nearly constant intensity from $\omega=30-90^{\circ}$. The close coincidence of the structural changes with increasing $\mathrm{CN}$ concentration seen in the R-SoXS (see Figure 4) and GIWAXS experiments reveals a close conjunction of mesoscale domain structure and crystal orientation. Since P(NDI2OD-T2) forms a fiber network in the Xy- cast film that is still present in the 5 vol.\% CN-cast blend but disappear for 10 vol.\% and larger $\mathrm{CN}$ concentrations, we propose that these fibers contain P(NDI2OD-T2) crystals with face-on orientation. On the other hand P(NDI2OD-T2) crystals are oriented edge-on in the circular domains which dominate the structure in the optimized blends. The $\mathrm{CN}$-induced orientation change is also observed in pristine P(NDI2OD-T2) films, ${ }^{[53]}$ which demonstrates that it is a solvent-controlled process that is also functional in the blend.

The second change appears to the P3HT crystal population. Here, higher CN concentrations introduce a significant fraction of randomly oriented crystals, while the edge-on preference is conserved. We note that the relative population of randomly oriented crystals is actually larger than it is implied by Figure 6, which can be seen after performing a geometrical correction of the raw pole figures (see Figure S12). After summing over all possible crystal angles, the corrected pole figures represent the total population distribution as a function of $\omega$. Integration of this curve directly yields the relative degree of crystallinity (DoC). However, the same conclusion can be drawn from these corrected pole figures, that in the optimized 
blends, P3HT and P(NDI2OD-T2) crystals adopt the same preferential edge-on orientation distribution, while they are almost perpendicularly oriented in the Xy-cast blend.

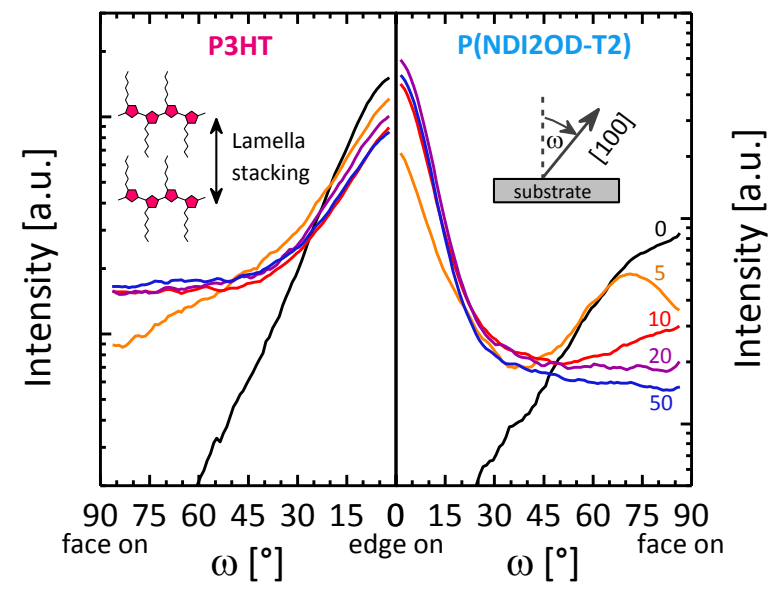

Figure 6 Pole figures of the [100] lamellar stacking of P3HT (left) and P(NDI2OD-T2) (right) crystals in the blend films. The $\mathrm{CN}$ concentration is indicated in the right figure. The definition of the polar angle $\omega$, as well as the investigated molecular stacking direction are given as inset.

Beside orientation, the crystal coherence and the DoC are influenced by the formulation of the casting solution. Figure 7 displays the change in P3HT and P(NDI2OD-T2) lamella coherence length for the various $\mathrm{CN}$ concentrations, which is comparable for both polymers. From the relatively large values of $12 \mathrm{~nm}$ for $\mathrm{P} 3 \mathrm{HT}$ and $18 \mathrm{~nm}$ for $\mathrm{P}(\mathrm{NDI} 2 \mathrm{OD}-\mathrm{T} 2)$, the coherence length jumps to about $30 \mathrm{~nm}$ and $45 \mathrm{~nm}$ for P3HT and P(NDI2OD-T2), respectively, when adding 5 vol.\% of $\mathrm{CN}$. More $\mathrm{CN}$ does not seem to affect the crystal coherence dimension. Note that these values are larger than what has been commonly reported for thermally annealed blends of polymers with soluble fullerenes and even larger than those reported for pure P3HT and P(NDI2OD-T2), which means that the high temperature drying procedure we use for the preparation of our active layers enables the growing of relatively large polymer crystals. ${ }^{[33,47,53,54]}$ The abrupt change of the crystal coherence for $\mathrm{CN}$-cast blends can be also seen in the evolution of the DoC of P3HT, which also doubles compared to the $\mathrm{Xy}$-cast blend but is constant above $5 \mathrm{vol} . \% \mathrm{CN}$ in the solution. Interestingly, the DoC of P(NDI2OD-T2) does not show this abrupt variation but instead decreases continuously. Since the bulk of the photocurrent enhancement occurs at $\mathrm{CN}$ concentrations above 5 vol.\% CN, these changes in DoC and lamellar coherence cannot explain the changes in device performance. 

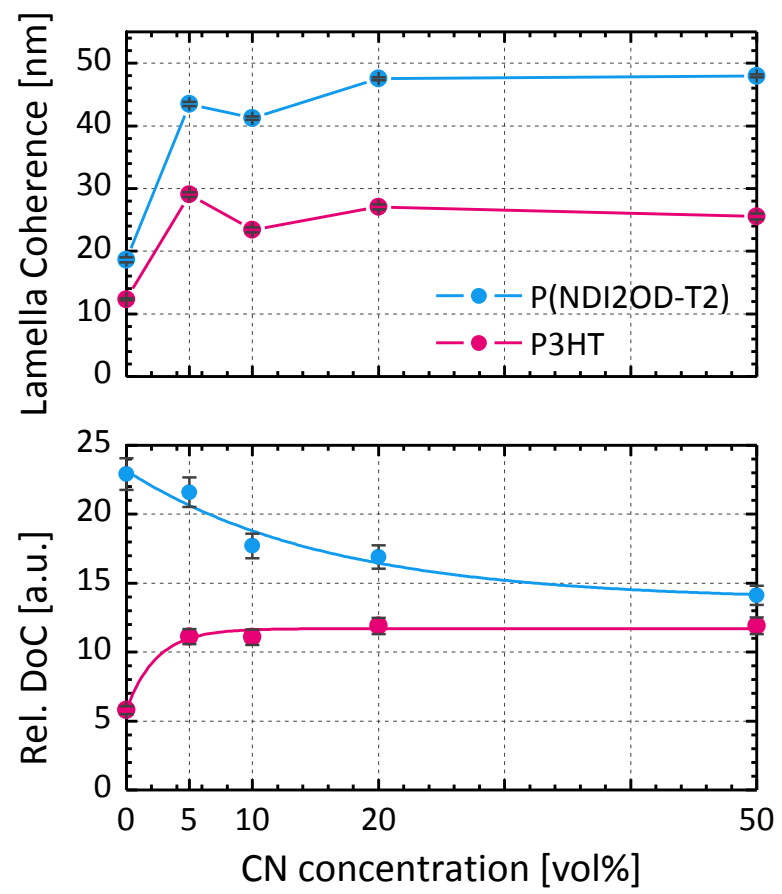

Figure 7 Lamella coherence (top) and relative DoC (bottom) of the different blends. Lines are guides to the eye. Uncertainties in the coherence are from the unweighted peak fits. Uncertainties in the relative $\mathrm{DoC}$ are derived from that of the illuminated sample volume.

\subsection{Steady-state exciton quenching}

From the investigation of the blend morphology, we next analyze the yields of excitons at the P3HT:P(NDI2OD-T2) heterojunction, which initiates the cascade of elementary processes leading to the generation of the photocurrent. The quenching (by charge-transfer) of excitons created after photon absorption in competition with their recombination to the ground state is simply probed by measuring the decrease of the photoluminescence (PL) of the donor (acceptor) in presence of an acceptor (donor). ${ }^{[55]}$ For P3HT, the singlet exciton quenching is almost complete and ranges from $92 \%$ to $96 \%$ (see Table 1). The high P3HT quenching for the micrometer large domains of the Xy-coated film is consistent with the observed low domain purity in the STXM experiment, while we assign the slightly increased quenching in the $\mathrm{Xy}: \mathrm{CN}$-processed films to the strongly reduced domain size. Determining the P(NDI2ODT2) PL quenching efficiency (PQE) is much more challenging due to the very low PL quantum efficiency of pure P(NDI2OD-T2), which we find is only about $0.14 \%$. In the 50 vol.\% CN-cast blend only $55 \%$ of the excitons can be quenched, while in the Xy-cast film the PQE is even lower and reaches $45 \%$. The incomplete quenching of the acceptor excitons therefore represents a direct and significant loss channel. Furthermore, we can safely state that 
the decreased IQE for photons absorbed in the acceptor is mainly caused by the incomplete quenching of the primary-excited excitons in P(NDI2OD-T2).

In combination with the knowledge of the domain structure, the exciton quenching results yield information about the composition of the donor and acceptor domains. In section 2.3 we observed that the P3HT-rich domains are larger than the P(NDI2OD-T2) domains in both the $\mathrm{Xy}$ - and 50:50 Xy:CN-cast films. Surprisingly, the PL quenching of P3HT is about twice that of P(NDI2OD-T2) and concentration-dependent PL quenching measurements shown in Figure S8 reveal that both polymers have a comparable exciton diffusion length. This result implies that a significant fraction of P(NDI2OD-T2) is dissolved in the P3HT-rich phase. On the other hand the poor quenching of P(NDI2OD-T2) even in the small domains of the 50:50 $\mathrm{Xy}: \mathrm{CN}$-cast blend, leads us to conclude that they are relatively pure and that the miscibility of $\mathrm{P} 3 \mathrm{HT}$ in the $\mathrm{P}(\mathrm{NDI} 2 \mathrm{OD}-\mathrm{T} 2)$ phase is rather low. This could be a consequence of a strong driving force for aggregation of P(NDI2OD-T2) during the transition from solution to the solid state ${ }^{[35,40]}$ tending to exclude P3HT from the domains. Thus, the incomplete quenching of $\mathrm{P}(\mathrm{NDI} 2 \mathrm{OD}-\mathrm{T} 2)$ excitons is a result of the high purity of the acceptor domains.

\subsection{Geminate recombination}

In the previous sections we have estimated that the IQE is significantly lower than the photoluminescence quenching over the entire spectral region, meaning that a process in the quantum efficiency cascade subsequent to exciton quenching must be the dominant loss mechanism for these devices as a whole. The next step in the cascade is the split-up of the geminate electron-hole pair that is created upon exciton quenching. In this section we will follow the change in photo-induced absorptions and bleaching as excitons move to the interface to create geminate charge carrier pairs, which subsequently create free charge carriers or decay to the ground-state. From the population flows on the early timescales initiated by photons absorbed in both the donor and in the acceptor, we obtain a holistic overview of the photophysical processes that limit the quantum efficiency in these blends.

\subsubsection{Transient absorption measurements}

We will first assign the spectral signatures of the different excited-state species contributing to the TAS spectrum. Figure 8.a shows the transient absorption of the pristine P(NDI2OD-T2) film after a delay of about $1 \mathrm{ps,}$, where the primary excitations are singlet excitons $(\mathrm{Ex}) .{ }^{[35]} \mathrm{A}$ 
negative change in transmission is observed in the NIR and visible spectral region, spanning the range from 1.25 to $1.55 \mathrm{eV}$ and 2.1 to $2.5 \mathrm{eV}$, respectively, which we attribute to the photo-induced absorption (PIA) of P(NDI2OD-T2) singlet excitons. The positive change in transmission on the other hand, which dominates the spectrum from 1.55 to $2.1 \mathrm{eV}$, is assigned to the ground state bleach (GSB) of neutral P(NDI2OD-T2) chromophores, which has a characteristic double peak structure (with maxima at 1.6 and $1.8 \mathrm{eV}$ ) which stems from the population of aggregate states. In our previous study of pristine P(NDI2OD-T2) we also described long-lived excitations (with ns lifetime) which showed only a ground state bleach but no photo-induced absorption. ${ }^{[35]}$ Using transient electron paramagnetic resonance spectroscopy (EPR) we are able to identify this states as P(NDI2OD-T2) triplet states that are formed in pristine films (Figure S11). The PIA signal of negative polarons $\left(\mathrm{P}^{-}\right)$, is obtained from charge accumulation spectroscopy (CAS) spectra, also shown in Figure 8.a. ${ }^{[56]}$ Two distinct PIA bands can be identified, ranging from 1.1 to $1.55 \mathrm{eV}$ and 2.5 to $3.0 \mathrm{eV}$. We note that the latter is well separated from the PIA of the excitons.

TAS and CAS spectra are also used to clarify the spectral range of possible TAS features of pristine P3HT, which are displayed in Figure 8.b. Negative transmission changes in the CAS spectra are assigned to the PIA of positive charges $\left(\mathrm{P}^{+}\right)$, which have a very broad and featureless spectrum ranging from 1.2 to $1.9 \mathrm{eV}$. Previous TAS measurements showed that also the singlet excitons contribute a negative signal that overlaps with the $\mathrm{P}+$ signal. $^{[57]}$ However, the Ex signal reaches a maximum at about $1.1 \mathrm{eV}$, as it is also apparent from Figure 8.b. ${ }^{[57,58]}$ According to the absorption spectra of aggregated, regio-regular P3HT, the positive GSB is observed for energies of $1.9 \mathrm{eV}$ and higher. ${ }^{[38,57]}$ In addition, the positive contribution of stimulated emission (SE), which overlaps with the $\mathrm{P}^{+}$and Ex PIA signals, is observed in the range of 1.35 to $1.9 \mathrm{eV} .^{[38,57]}$

Figure 8.c shows the evolution of the TAS spectra for the 50 vol.\% $\mathrm{CN}$-cast blend within the first nanosecond, recorded upon selective excitation of P(NDI2OD-T2) at $1.51 \mathrm{eV}$. A first inspection reveals that the kinetics of the acceptor GSB in the blend evolves more rapidly than in the pristine layer, and that the bleach signal after $1 \mathrm{~ns}$ is rather faint. This demonstrates that the efficiency for forming long-lived species (charges or triplet excitons) is low, as we will discuss in the following section. 


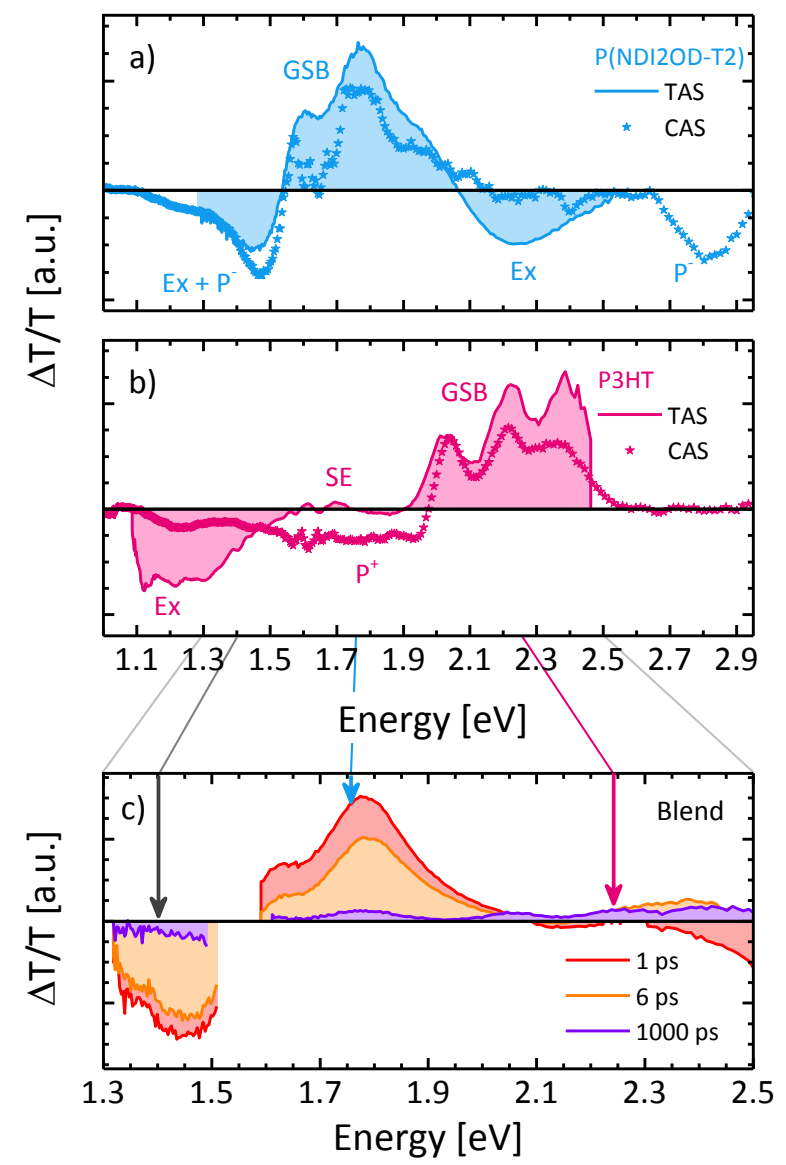

Figure 8 Transient absorption spectroscopy (TAS) and charge accumulation spectroscopy (CAS) of a) pristine $\mathrm{P}(\mathrm{NDI} 2 \mathrm{OD}-\mathrm{T} 2)$ and $\mathbf{b})$ pristine $\mathrm{P} 3 \mathrm{HT}$. Excitons $(\mathrm{Ex})$ and polarons $(\mathrm{P})$ contribute a negative photo-induced absorption signal, while the ground-state bleach (GSB) and stimulated emission (SE) give a positive change in transmission. c) TAS spectra of the 50 vol.\% $\mathrm{CN}$-cast blend after selective excitation of P(NDI2OD-T2) at $1.51 \mathrm{eV}$. For further analysis, the dynamics within narrow energy intervals (indicated by the arrows) are shown in Figure 9.

\subsubsection{Geminate pair formation and recombination}

In Figure 9 we display the transient absorption kinetics observed for the 50 vol. $\% \mathrm{CN}$-cast blend. At the beginning of the experiment, P(NDI2OD-T2) excitons were selectively excited at an energy of $1.51 \mathrm{eV}$. The change of the transient transmission is then analyzed at the energy of $2.25 \mathrm{eV}$ (called "P3HT bleach region"). Here, only the positive GSB of P3HT and the negative PIA of $\mathrm{P}(\mathrm{NDI} 2 \mathrm{OD}-\mathrm{T} 2)$ excitons contribute to the signal. Thus, as the $\mathrm{P}(\mathrm{NDI} 2 \mathrm{OD}-\mathrm{T} 2)$ excitons move to the interfaces and form charge carrier pairs, the signal in this region should go from negative to positive. Indeed this is what we observe in Figure 9.a in the time region marked A. In the first few picoseconds the signal in this region changes from negative to positive as the PIA of P(NDI2OD-T2) excitons that are quenched is replaced by the P3HT absorption bleach of the resultant holes. Fitting the rise of P3HT bleach yields 
the violet line and a lifetime for the P(NDI2OD-T2) in the blend of $0.96 \pm 0.04 \mathrm{ps}$, about 40 times faster than the luminescence lifetime of pristine P(NDI2OD-T2). ${ }^{[35]}$
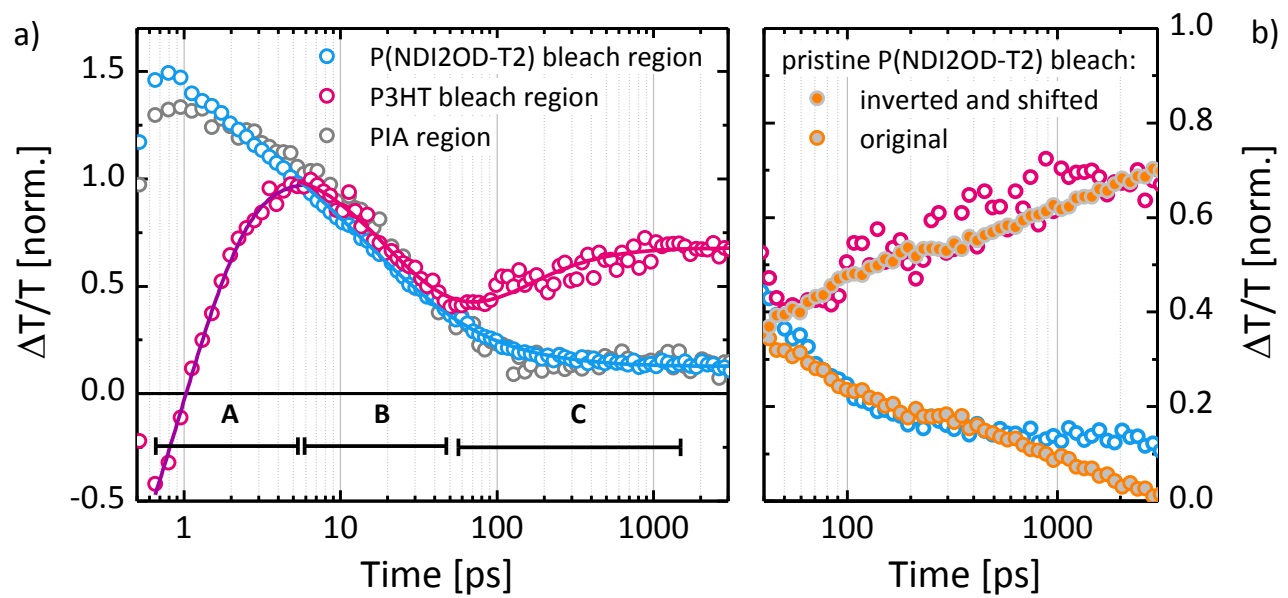

Figure 9 (left) Evolution of transient absorption spectra at $2.25 \mathrm{eV}$ (P3HT bleach region), $1.75 \mathrm{eV}$ (P(NDI2OD-T2) bleach region) and $1.4 \mathrm{eV}$ (PIA region) in the 50 vol.\% CN-cast blend after selective excitation of $\mathrm{P}(\mathrm{NDI} 2 \mathrm{OD}-\mathrm{T} 2)$ at $1.51 \mathrm{eV}$. The signals are normalized to their value when charge carrier generation is complete (end of rise in $\mathrm{P} 3 \mathrm{HT}$ bleach in time region A). The kinetics are overlaid with the fit described in the text. (right) Comparison of the bleach of the blend components to the kinetics of pristine P(NDI2OD-T2) (inverted and offset) in time region C.

Now following the kinetics of this region further ahead in time we see that the signal decreases in the time range marked $\mathrm{B}$, which can clearly be explained by a loss of P3HT bleach due to fast charge carrier recombination. This ultrafast geminate recombination is followed by an increase of the intensity in time region $\mathrm{C}$. We attribute this increase of the intensity to the gradual recombination of long-lived P(NDI2OD-T2) excitons, that do not reach the heterojunction. More precisely, the decreasing (negative) PIA of P(NDI2OD-T2) excitons and the constant (positive) GSB of P3HT holes result in a total signal that increases with time. To support this assumption, we show in Figure 9.b the kinetics of the decay of long-lived P(NDI2OD-T2) excitations measured in the pristine polymer. If we invert and shift these kinetics, we can see that they reproduce well the kinetics in region $\mathrm{C}$. This result suggests that there are two populations of P(NDI2OD-T2) excitons, one which promptly generates geminate charge carrier pairs which transfers with an inverse rate of around 1 ps (in time region $\mathrm{A}$ ), and one that is much longer lived and accounts for the P(NDI2OD-T2) emission observed under steady-state illumination as well as for the increase in the P3HT bleach in time region $\mathrm{C}$. We note that the observation of long-lived P(NDI2OD-T2) excitons is in agreement with the low PQE of P(NDI2OD-T2), that was presented above.

The data in Figure 9.a also allow to estimate a lower bound for the percent of long-lived free charge carriers. For that, we can look at the kinetics at the energy of $1.8 \mathrm{eV}$ (P(NDI2OD-T2) bleach region), where the signal is composed of the positive P(NDI2OD-T2) GSB (either 
excitons or electrons) and the negative PIA of positive charges on P3HT. Normalizing these signals to the value when the conversion of excitons to geminate pairs is complete (at $5 \mathrm{ps}$ ), and assuming that charges are the only species that causes the subsequent decay of the signals in the P3HT and P(NDI2OD-T2) bleach regions, then the value at long time would reflect the signal that is related to long-lived, free charge carriers. By looking at the data, this would give an estimate of $\sim 15 \%$. Now we know that some of the signal decay in these regions is actually due to decay of the long-lived P(NDI2OD-T2) excited-states, we conclude that at least $15 \%$ of charge carriers are long-lived.

In order to get a better estimate of the fraction of free charge carrier and to demonstrate the validity of our assumptions, the kinetics were fit with a global model. Here we take into account charge carriers that can either be short or long-lived, and long-lived P(NDI2OD-T2) excitons. Using stretched exponentials to parameterize the recombination of short-lived charge carriers and the long-lived P(NDI2OD-T2) excitons we can write $\Delta T / T_{i}=A_{i}\left(p_{1 a} e^{-\left(t / \tau_{1}\right)^{b_{1}}}+p_{1 b}\right)+B_{i} e^{-\left(t / \tau_{2}\right)^{b_{2}}}$; where $A$ and $B$ are constants relating to the splitting of the population into two species (charges and excitons) and the absorption cross sections of the charge carriers in each wavelength range. The remaining parameters are shared between the wavelength regions. Here, $p_{1 a}$ represents the fraction of the charge carriers that are shortlived and recombine with a rate described by $\tau_{1}$ and $b_{1}, p_{1 b}$ represents the fraction of charge carriers that are long-lived (e.g. potentially useful in the solar cell), while $\tau_{2}$ and $b_{2}$ represent the decay of the P(NDI2OD-T2) excitons. The results of this global fit are shown as the pink and blue lines in Figure 9.a, and the parameters for both fits are given in Table 2. We find that $p_{1 b}$ is approximately 0.3 , so only roughly $30 \%$ of the charge carriers formed are longlived, while $70 \%$ of the charge carriers recombine geminately on the 100 picosecond timescale, far too fast to produce photocurrent. We note that the kinetics in the P(NDI2ODT2) bleach region perfectly resembles the kinetics in the PIA region at $1.4 \mathrm{eV}$, where all excited states contribute to the signal, implying that our model is able to describe all relevant processes quantitatively.

Combining these results with the steady state quenching observations we can approximate the upper bound of the IQE in the P(NDI2OD-T2) phase as 0.50 (due to incomplete quenching) $\times$ 0.30 (due to geminate recombination), which yields an estimate of 0.15 . This is still roughly a factor of 2 larger than the observed IQE for P(NDI2OD-T2) excitation, indicating perhaps that not all the long-lived charge carriers can be extracted. Nonetheless, we see that the 
combination of incomplete quenching and fast geminate recombination explain the majority of the quantum efficiency loss after excitation of P(NDI2OD-T2).

Table 2 Parameters extracted from fitting the data in Figure 9.a.

\begin{tabular}{|c|c|c|c|c|c|c|c|c|}
\hline & $p_{1 a}$ & $p_{1 b}$ & $\begin{array}{c}\tau_{1} \\
{[\mathrm{ps}]}\end{array}$ & $b_{1}$ & $\begin{array}{c}\tau_{2} \\
{[\mathrm{ps}]}\end{array}$ & $b_{2}$ & $A$ & $B$ \\
\hline P3HT bleach region & $0.7 \pm 0.1$ & $0.3 \pm 0.1$ & $17 \pm 2$ & $0.8 \pm 0.1$ & $8 \pm 4$ & $0.4 \pm 0.1$ & $2.2 \pm 0.4$ & $-3.3 \pm 0.7$ \\
\cline { 1 - 8 } $\begin{array}{c}\text { P(NDI2OD-T2) bleach } \\
\text { region }\end{array}$ & & & $0.41 \pm 0.1$ & $1.3 \pm 0.3$ \\
\hline
\end{tabular}

Transient absorption spectra were also measured upon excitation of P3HT. The results are displayed in Figure S9 and reveal that excitons on the P3HT are quenched within about 10 ps. Unfortunately, the shifting of the different excited states species between ordered and disordered regions complicate the analyses of the dynamics of these spectra. Furthermore, a possible energy transfer from P3HT to $\mathrm{P}(\mathrm{NDI} 2 \mathrm{OD}-\mathrm{T} 2)$ may also influence the kinetics, although we have no indications that this transfer is very efficient. However, one can again use the signal at $1.4 \mathrm{eV}$ as a probe for the decay of geminate charge carrier pairs. Figure S10 shows that after quenching of the excitons, again an fast geminate recombination destroys the majority of excited states within less than 200 ps and that the signal decreases to a value of about $15 \%$ of the original intensity. Thus, the carrier dynamics are broadly similar upon P3HT or P(NDI2OD-T2) excitation, which finally reveals that geminate recombination losses on the order of $70 \%$ (as suggested by the steady state excitation of the CTS) are reasonable, and that we can directly observe the sub-nanosecond recombination that leads to these losses.

\section{Discussion and Conclusions}

Based on the above studies of structure and excited state dynamics, Figure 10 sketches the interplay of morphology and charge generation in the best performing P3HT:P(NDI2OD-T2) blend processed from a 50:50 Xy:CN mixture. In Figure 10.a a schematic overview of the domain structure, reconstructed from the sulphur map and R-SoXS profile, is shown. It provides a detailed overview of the most important morphological and structural features, which dominate the charge generation process, as we will discuss in the following. 

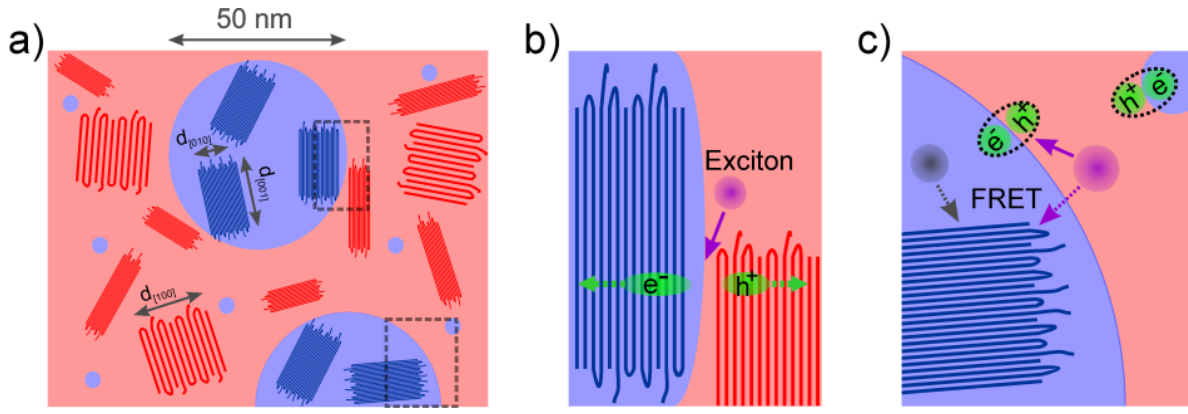

Figure 10 a) Schematic representation of the morphology of the 50:50 Xy:CN-cast blend. The image illustrates a cross section in-plane with the substrate (top view), showing P(NDI2OD-T2) domains (blue) and the P3HT matrix (red). Ordered regions with darker colors represent the polymer backbones in crystalline domains, where face-on crystals have a 5 times larger lattice plane spacing than edge-on crystals. Small P(NDI2OD-T2) enclosures, which have the size of a single aggregate, ${ }^{[35]}$ account for the lower purity of the P3HT domains. b) Face-to-face donor/acceptor orientation at the heterojunction. This geometry is proposed to facilitate free charge carrier generation. c) Effect of exciton self-trapping in P(NDI2OD-T2) aggregates. Excitons from disordered acceptor or donor regions can transfer to aggregated or crystalline P(NDI2OD-2) regions by Förster-resonant energy transfer (FRET). In addition, amorphous donor/acceptor interfaces are illustrated, were geminate pairs undergo fast recombination.

In order to identify the factors dominating the free charge carrier yield in P3HT:P(NDI2ODT2) blends, we tried to correlate the photocurrent of the solar cell devices from Figure 1.a to the domain size, the domain purity and to the size and DoC of the polymer crystallites. However, the comparison of the dependence of the PL quenching (see Tab. 1), the TSI (see Figure 4.b), the DoC (see Figure 7.a) and the coherence length (see Figure 7.b) to the photocurrent reveal that these parameters all show either a marginal change over the whole $\mathrm{CN}$ concentration or an abrupt change upon addition of only 5 vol. $\% \mathrm{CN}$ followed by more or less constant value. The only parameter that mimics the continuous change of the photocurrent is the redistribution of the $\mathrm{P}(\mathrm{NDI} 2 \mathrm{OD}-\mathrm{T} 2)$ crystal, as it is evident from the pole figure in Figure 6. Note that in a recent study, Fabiano and co-workers also observed a perpendicular orientation of the P3HT and P(NDI2OD-T2) crystallites in low performing devices prepared from xylene and dichlorobenzene, while the best performing cells cast from $\mathrm{Xy}: \mathrm{CN}$ reveal a parallel edge-on orientation of the polymer crystals. ${ }^{[59]}$ In addition, a higher dissociation rate was also reported at a planar heterojunction for zinc phthalocyanine donor molecules that are oriented face-on with respect to the acceptor domain compared to the edgeon configuration. ${ }^{[60]}$ Thus, we investigated the orientation of the polymer crystallites relative to each other in more details.

We start our discussion from the orientation distributions in the Xy-cast blend. Here, the donor and acceptor crystals are oriented almost perpendicular to each other. Addition of the co-solvent $\mathrm{CN}$ re-orients the originally face-on $\mathrm{P}(\mathrm{NDI} 2 \mathrm{OD}-\mathrm{T} 2)$ distribution to a 
predominantly edge-on orientation. At the same time, when the P(NDI2OD-T2) crystals start to adopt an orientation distribution that is similar to that of the P3HT polymer crystallites, the photocurrent also increases. Obviously, the highest photocurrents are observed for blends were $\mathrm{P} 3 \mathrm{HT}$ and $\mathrm{P}(\mathrm{NDI} 2 \mathrm{OD}-\mathrm{T} 2)$ have comparable orientation distributions comprising a strong edge-on preference. Thus, we make the assumption that not absolute crystal orientation but rather the face-to-face relative orientation of P3HT and P(NDI2OD-T2) crystallites represents the configuration in which efficient dissociation of charge-transfer states is possible. To demonstrate this correlation more quantitatively, we first calculated the relative DoC of P(NDI2OD-T2) edge-on crystals, which we obtained by counting all crystals with a polar angle smaller than $15^{\circ}$ (this is proportional to the area under the corrected pole figures from $\omega=0$ to $15^{\circ}$ ). Figure 11 displays the relative population of edge-on oriented P(NDI2OD-T2) crystals, plotted against the $J_{\mathrm{SC}}$ of the solar cells. The plot reveals the gradual increase of the photocurrent upon the generation of more and more edge-on oriented P(NDI2OD-T2) crystals.

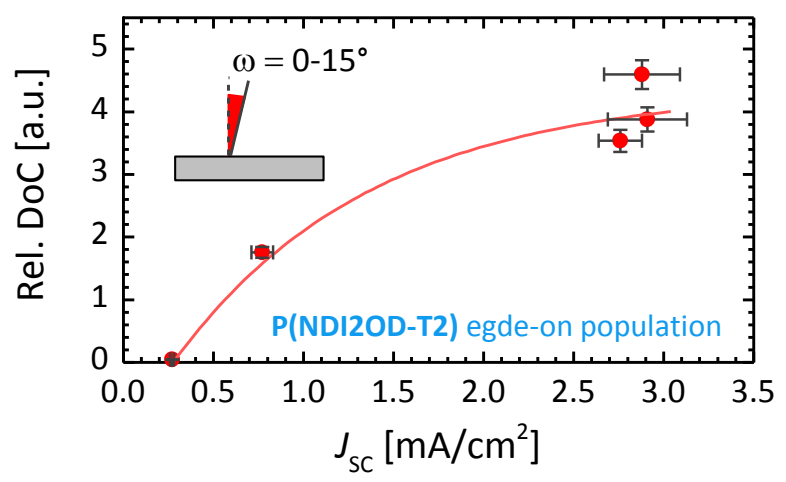

Figure 11 Correlation of the fraction of the P(NDI2OD-T2) edge-on crystal population with the short circuit current of the solar cell devices. DoC uncertainties derived from that of the illuminated sample volume, and $\mathrm{J}_{\mathrm{SC}}$ uncertainties derived from device statistics.

In order to understand the role of polymer crystals in the charge generation process it is instructive to reflect on some recent models aiming to describe photocurrent generation at organic donor/acceptor heterojunctions under consideration of the semi-crystalline nature of the materials. It has been noted that efficient carrier generation at distributed heterojunctions requires an unidirectional flow of carriers away from the heterojunction. ${ }^{[14,48,49]}$ The driving force behind that is supposed to originate from an energy gradient of the potential energy pointing from the heterojunction into the bulk of the pure phases. Recent experimental and theoretical results suggest that this gradient is introduced by the difference in ionization energy or electron affinity caused by the larger disorder in regions close to the heterojunction compared to better ordered or perfectly crystalline regions in the bulk. ${ }^{[10,48,49,61-63]}$ The decrease of the band gap in the ordered relative to the disordered phases can be strongly 
enhanced by the formation of intermolecular excited states, so called aggregates, as it has been described for P3HT, which lowers the band gap by about $0.35 \mathrm{eV} .^{[64,65]}$ For pure $\mathrm{P}(\mathrm{NDI} 2 \mathrm{OD}-\mathrm{T} 2)$, we demonstrated the strong tendency to form such aggregate states, which have a, by $0.3 \mathrm{eV}$, lower band gap compared to intra-chain excitons on disordered $\mathrm{P}(\mathrm{NDI} 2 \mathrm{OD}-\mathrm{T} 2)$ segments. ${ }^{[35]}$ A consequence of such a microscopic energy landscape is that charges as well as excitons are repelled from the heterojunction, which suppresses the recombination of free charges but also prevents the charge transfer of excitons directly at the interface. Instead, exciton dissociation requires that charge carriers are transferred over several nanometers. ${ }^{[48,66]}$ This can be mediated by tunnelling through the potential well, which results in a separated electron-hole pair with reduced binding energy. Very recently, Troisi calculated the distance over which the rate for exciton dissociation exceeds the recombination rate. This distance critically depends on the coupling between neighbouring hopping sites, which is orders of magnitude larger along the polymer backbone and pistacking direction than in the lamella-stacking direction. ${ }^{[66]}$ Thus, the face-to-face donor/acceptor orientation found in the optimized solar cells is favourable with respect to the tunnelling assisted exciton dissociation mechanisms described by Troisi and co-workers. In this case, both polymer crystals stand edge-on which exposes the polymer backbone towards the heterojunction. Figure 10.b illustrates this situation where e.g. an electron tunneling out of a P3HT crystal can easily reach the P(NDI2OD-T2) backbone within a crystalline acceptor domain. Note that the two crystals must not be in direct contact to each other. This process will be largely suppressed if the P(NDI2OD-T2) crystals are oriented face-on to the substrate, as the bulky side chains, now oriented towards the heterojunction, will constitute an additional tunneling barrier. Alternatively, if the CTS is indeed the precursor for all free charge carriers it is expected that the formation of coplanar crystals will also improve the efficiency for the CTS split-up, due to the larger delocalization within the crystals, according to the simulations of Tamura and Burghardt. ${ }^{[19,67,68]}$

The fact that not only the crystallinity but also the relative orientation must be optimized to enable optimal charge separation might display a special problem of conjugated polymers which usually carry long, insulating alky chains. In contrast, fullerenes are spherical symmetrical objects with no or relatively short side chains which have no preferential orientation with respect to the donor molecules. In addition, electrons can delocalize efficiently over neighboured fullerene molecules, ${ }^{[19,67]}$ and may therefore perform better than any other class of acceptor materials. 
At the end, we point out that the energy gradients between amorphous and ordered regions also affect the quantum efficiency in a negative way. Due to the large potential difference between amorphous and crystalline phases, excitons quickly migrate to the ordered, low energy states, as it is illustrated in Figure 10.c. We found that the spectral diffusion into these low energy states occurs on a timescale of about $10 \mathrm{ps}$ in pristine P(NDI2OD-T2), ${ }^{[35]}$ meaning that it competes with exciton quenching and the subsequent formation of charge-separated states. Once an exciton is generated on or transferred to a P(NDI2OD-T2) aggregate that is surrounded by amorphous content, it is restricted to exist deep within the ordered region. In effect, this has similar consequences to the phenomenon known as exciton self-trapping observed in molecular aggregates. ${ }^{[69]}$ Recently, Mori and co-workers demonstrated an efficiency record for all-polymer solar cells of $4.1 \%$, by combining the P(NDI2OD-T2) acceptor with a new donor copolymer, which demonstrate the great potential of P(NDI2ODT2) as an acceptor for organic solar cells. ${ }^{[70]}$ Similar to our results, the PL quenching of the $\mathrm{P}(\mathrm{NDI} 2 \mathrm{OD}-\mathrm{T} 2)$ was found to be significantly smaller than the PQE of the donor. Thus, relatively pure and crystalline acceptor domains may improve the charge carrier separation but then may also suffer from imperfect exciton quenching.

In summary, we propose a microscopic framework in which efficient charge carrier generation at organic heterojunctions can be realized. A proper orientation of the crystalline polymer domains with respect to the heterojunction is found to be a prerequisite in order to dissociate electron-hole pairs into free charge carriers efficiently. However, for very anisotropic systems, which conjugated polymers are in general, the correlated orientation of donor and acceptor crystals at the heterojunction is a challenging task, which requires a much larger control over the nanometer morphology, the degree of crystallinity, as well as of the crystal orientation. Compared to fullerenes, our results provide a reasonable explanation for the generally lower quantum efficiencies of polymeric acceptors. Further experimental and theoretical studies will be needed to examine the reasons for the importance of crystalline domains on the charge carrier dissociation process.

\section{Experimental}

\section{Materials}

$\mathrm{P}(\mathrm{NDI} 2 \mathrm{OD}-\mathrm{T} 2)$ has been synthesized following a recently described procedure. ${ }^{[24,40]} \mathrm{P} 3 \mathrm{HT}$ (BASF Sepiolid P200, purchased from Rieke Metals) was used as received. The molecular weights of P(NDI2OD-T2) and P3HT are $M_{\mathrm{n}} / M_{\mathrm{w}}=26.200 / 85.200 \mathrm{gmol}^{-1}$ and $M_{\mathrm{n}} / M_{\mathrm{w}}=$ $12.800 / 22.500 \mathrm{gmol}^{-1}$, respectively. Compared to our previous publication, ${ }^{[40]}$ the actual P3HT batch has a significantly lower molecular weight (12.800 $\mathrm{gmol}^{-1}$ compared to 
17.500 $\mathrm{gmol}^{-1}$ ), which might explain the lower photocurrents in the present study. We note that for all measurements presented here, we used the same batch of P3HT and P(NDI2ODT2) (except for the PDS measurements), to eliminate any batch- or molecular weight-related influences on the results. Solvents were purchased from Sigma Aldrich (CF, Xy, CB, DCB) or Alfa Aesar (85\% 1-chloronaphthalene with 15\% 2-chloronaphthalene).

\section{Solar cell preparation and characterisation}

Pre-structured, indium tin oxide (ITO) coated glass substrates were used for solar cell preparation. PEDOT:PSS (AI4083, $30 \mathrm{~nm})$ and Samarium(20 nm)/Aluminium(100 nm) were used as hole and electron collecting contacts. The final structure of the cells was Glass/ITO/PEDOT:PSS/Active layer/Sm/Al and the active area of the cells $16 \mathrm{~mm}^{2}$. Device preparation, transport and measurements were performed under inert atmosphere. Films that were prepared from a $\mathrm{CN}$-containing solution were spin-coated for $5 \mathrm{~s}$ and subsequently transferred to a hot plate where they finally dried. The active layer thickness was determined with a Dektak profilometer.

Solar cells where characterized under simulated sunlight of a Newport Oriel Sol2A solar simulator. Calibration of the setup was done with a KG5 filtered reference silicon solar cell (calibrated by Fraunhofer ISE) and the intensity was set to $100 \mathrm{~mW} \mathrm{~cm}^{-2}$. The irradiance of the solar simulator was monitored before each measurement by a calibrated KG5 filtered solar cell which is directly integrated into the home build, temperature controlled sample holder, with the temperature set to $20{ }^{\circ} \mathrm{C}$.

For EQE measurements, light from a $100 \mathrm{~W}$ quartz halogen lamp (Philips 7724) was timemodulated by a mechanical chopper $(90-140 \mathrm{~Hz})$ and fed into a Cornerstone 260 1/4m monochromator (model 74100). The monochromatic output was focused onto a fibre to transfer the light into the glovebox. The modulated photocurrent from the solar cell was detected over the input resistor of a lock-in amplifier (Princeton Research Instruments). To increase the sensitivity of the setup, an input resistance of $1 \mathrm{k} \Omega$ was used. No white light background illumination was applied. For IQE calculations in Figure 1.c, the absorption $A$ was estimated from the measured optical density of the films and it was assumed that light passes twice the active layer thickness, which is a reasonable approximation for the thick films used for our devices. The spectra were not corrected for reflection losses.

\section{PL quenching}

Films for PL quenching measurements are prepared on uncovered glass slides, as we found that PEDOT:PSS or PSS significantly reduce the PL of the polymers. To calculate PL 
quenching efficiency, the PL efficiency of blend samples is compared to that of the pure polymers. Because PL efficiency of the pure polymers also varies for the different preparation conditions (e.g. drying temperature, solvents), a single donor and acceptor reference sample was prepared for each blend sample that was studied. For pure P3HT and P(NDI2OD-T2), we find quantum efficiencies of $7-12 \%$ and $0.09-0.2 \%$, respectively. Measurements are performed using an integrating sphere setup (Hamamatsu C9920-02, A10094, C10027). For excitation, the EQE setup was used (see above). PL quenching of P3HT and P(NDI2OD-T2) are probed by excitation at $500 \mathrm{~nm}$ and $700 \mathrm{~nm}$, respectively, and the entire PL spectrum up to $950 \mathrm{~nm}$ was integrated to obtain the number of emitted photons. Determination of the $\mathrm{P}(\mathrm{NDI} 2 \mathrm{OD}-\mathrm{T} 2)$ quenching was limited by the low PL quantum yield of P(NDI2OD-T2). To enhance the sensitivity of the setup, an optical density (OD) filter (OD 0.8-1.5) was used during measurement of the excitation intensity, which was removed when the PL of the films was measured. The true excitation intensity was calculated afterwards, using the measured optical density of the filter. All steps, including film preparation, sample transport and measurement were performed under inert atmosphere, as films which have been exposed to oxygen show strongly reduced PL efficiencies (up to a factor of 2).

\section{Transient absorption spectroscopy}

Transient absorption (TA) measurements were performed with a home-built pump-probe setup. To measure in the time range of $1 \mathrm{ps}$ to $4 \mathrm{~ns}$ with a resolution of $\sim 100 \mathrm{fs}$, the output of a commercial titanium:sapphire amplifier (Coherent LIBRA HE, $3.5 \mathrm{~mJ}, 1 \mathrm{kHz}, 100 \mathrm{fs}$ ) was split into two beams independently pumping two optical parametric amplifiers (Coherent OPerA Solo). One optical parametric amplifier (OPA) was used to generate the $530 \mathrm{~nm}$ excitation pulse, the other OPA was used to generate a $1300 \mathrm{~nm}$ seed pulse for white-light generation with a sapphire window in the visible. For excitation with $800 \mathrm{~nm}$ the direct output of the LIBRA was used. The variable delay of up to $4 \mathrm{~ns}$ between pump and probe was introduced by a broadband retroreflector mounted on a mechanical delay stage. Only reflective elements were used to guide the probe beam to the sample to minimize chirp. The excitation pulse was chopped at $500 \mathrm{~Hz}$, while the white light pulses were dispersed onto a linear photodiode array which was read out at $1 \mathrm{kHz}$. Adjacent diode readings corresponding to the transmission of the sample after an excitation pulse and without an excitation pulse were used to calculate $\Delta \mathrm{T} / \mathrm{T}$. TA measurements were performed at room temperature under dynamic vacuum at pressures lower than $10^{-5}$ mbar.

\section{Photothermal deflection spectroscopy (PDS) and IQE}


For the PDS measurements monochromated light from a $100 \mathrm{~W}$ QTH lamp was chopped $(3.333 \mathrm{~Hz})$ and focused onto a photovoltaic active layer spincoated onto a quartz substrate. The temperature change caused by the de-excitation of the absorbed light was probed by immersing the sample in a deflection medium (Perfluorohexane, 3MFluorinert FC-72). The change in refractive index caused by the heating of the medium is probed by a HeNe laser $(633 \mathrm{~nm})$ of which the deflection is detected by a position-sensitive Si detector, connected to a Stanford Research Systems SR830 lock-in amplifier. The PDS spectra were set to absolute scale by matching the spectra with integrating sphere measurements using a Varian Cary 5000 spectrophotometer. The fraction of absorbed photons by the active layer in solar cell configuration in the weakly absorbing subgap region was calculated by the simplified assumption of the incoming light waves passing through the active layer twice owing to reflection from the metallic cathode.

\section{Energy-filtered transmission electron microscopy}

TEM measurements were performed with a Libra 200 FE (Carl Zeiss Microscopy GmbH) equipped with an in-column spectrometer. The primary electron energy was set to $200 \mathrm{keV}$. Energy-filtered (EF-) TEM images were recorded near the sulphur ionization edge (165 eV energy loss) using an energy window of $10 \mathrm{eV}$. Elemental maps were generated with the ImageJ software package and EFTEMj plugin. ${ }^{[71]}$ The background was extrapolated with a power-law model from pre-edge images (acquired at 130, 140, $150 \mathrm{eV}$ for Figure 3.a and at 110, $140 \mathrm{eV}$ for Figure 3.b) and subtracted from the post-edge image (195 eV). Prior to processing, sub-images were carefully aligned using reference images and the TurboReg plugin for ImageJ to compensate drift and shrinking of the specimen during the measurement. ${ }^{[72,73]}$ The nominal resolution (defined by the image resolution) of the EF-TEM maps in Figure 3.a and b is 0.08 and 0.2 pixel/nm, respectively.

\section{$X$-ray samples}

Samples for X-ray measurements were prepared in parallel to solar cells fabrication from the same solutions and with identical protocols. To reduce the background scattering in GIWAXS, Si wafers covered with a thin PSS (Poly(sodium 4-styrenesulfonate) aqueous solution, $4 \mathrm{wt} \%$, Sigma Aldrich) layer served as substrate. For transmission experiments (scattering and microscopy), fragments of the same film were floated off the substrate by dissolving the PSS layer in water. For R-SoXS, the films were picked up from the water with $100 \mathrm{~nm} \mathrm{SiN}$ windows (Norcada), while films were picked up with copper TEM grids (300 mesh, Gilder) for STXM measurements.

\section{Resonant scattering and scanning transmission X-ray microscopy}


R-SoXS measurements were performed at the 11.1.0.2 beamline of the Advanced Light Source (ALS) ${ }^{[74]}$ The incident beam size of $(200 \mu \mathrm{m} \times 150 \mu \mathrm{m})$ was set by collimating slits. The scattering pattern was captured by a Princeton PI-MTE CCD cooled to $-45^{\circ} \mathrm{C}$. For the full q-range, scattering patterns were acquired with two sample-detector distances at $190 \mathrm{~mm}$ and $50 \mathrm{~mm}$. A modified version of the NIKA software package was used to average the data into profiles. STXM measurements were performed at the 5.3.2.2 beamline of the ALS. ${ }^{[75]}$ Composition and thickness maps were extracted from the images acquired at two energies using the same procedures as previously published. ${ }^{[76]}$

\section{Grazing-incidence wide-angle $X$-ray scattering}

Measurements were performed at the 7.3.3 Beamline of the ALS (Beam energy at $10 \mathrm{keV}$ and beam width $\sim 1 \mathrm{~mm}) .{ }^{[77]}$ A Pilatus $1 \mathrm{M}$ photon-counting detector array was used to measure diffraction intensities. Most of the x-ray flight path was contained by a Helium atmosphere to reduce air scatter in the signal. The edges of the substrates were cleaved to eliminate diffraction signal from the edge of the spincast films, leaving approximately the center $1 \mathrm{~cm} \mathrm{x}$ $1 \mathrm{~cm}$ of the film. Diffraction intensities were acquired at an incident angle just above the film critical angle for maximum diffraction signal and an incident angle well above the critical angle of both film and the substrate $\left(\alpha=0.20^{\circ}\right)$ where intensities are linear to illuminated film volume. The results from analysis of the measurements at critical angle are presented here with their intensities scaled to those measured at the high incident angle. The intensities are additionally corrected for beam footprint (length of the sample in the beam) and film thickness.

Pole figures were constructed by integrating the intensities at each detector azimuth within a q-range encompassing the diffraction peak and subtracting off a linear background defined by the intensities at either end of the integrated q-range. The intensities are presented as a function of polar angle on the crystallographic orientation sphere $(\omega)$ after conversion from the detector azimuth using the known scattering geometry. The relative degree of crystallinity (DoC) was calculated from these pole figures by integrating the intensities over the crystallographic orientation sphere such that $\mathrm{DoC}=\int I(\omega) \sin \omega d \omega$. For measurement of crystal spacing and coherence length, peak functions were fit to profiles I(q) averaged over the detector azimuth. The coherence length $(2 \pi / \mathrm{FWHM})$ is calculated from the first order diffraction peaks. Disorder or instrumental broadening of the peaks were not considered here.

\section{Supporting Information}

Supporting Information is available online from the Wiley Online Library or from the author. 


\section{Acknowledgements}

The authors thank Oleksandr V. Mikhnenko and Markus Wollgarten for helpful discussions. We specifically thank Thuc-Quyen Nguyen for providing the DPP(TBFu $)_{2}$, Riccardo Di Pietro, Iyad Nasrallah and Henning Sirringhaus for providing the CAS spectra and Burkhard Stiller for performing the SKPM measurements. M.S. acknowledges financial support from the German Science Foundation (DFG) within the priority program SPP 1355. D.N. acknowledges a DFG travel grant. B.A.C. acknowledges financial support of the NIST-NRC postdoctoral fellowship program. The Advanced Light Source is supported by the Director, Office of Science, Office of Basic Energy Sciences, of the U.S. Department of Energy under Contract No. DE-AC02-05CH11231.

\section{References}

[1] Z. He, C. Zhong, S. Su, M. Xu, H. Wu, Y. Cao, Nat. Photonics 2012, 6, 591.

[2] S. H. Park, A. Roy, S. Beaupre, S. Cho, N. Coates, J. S. Moon, D. Moses, M. Leclerc, K. Lee, A. J. Heeger, Nat. Photonics 2009, 3, 297.

[3] J. Peet, J. Y. Kim, N. E. Coates, W. L. Ma, D. Moses, A. J. Heeger, G. C. Bazan, Nat. Mater. 2007, 6, 497.

[4] T. Kietzke, H. H. Horhold, D. Neher, Chemistry of Materials 2005, 17, 6532.

[5] X. M. He, F. Gao, G. L. Tu, D. Hasko, S. Huttner, U. Steiner, N. C. Greenham, R. H. Friend, W. T. S. Huck, Nano Lett. 2010, 10, 1302.

[6] E. J. Zhou, J. Z. Cong, Q. S. Wei, K. Tajima, C. H. Yang, K. Hashimoto, Angew. Chem.-Int. Edit. 2011, 50, 2799.

[7] D. Mori, H. Benten, H. Ohkita, S. Ito, K. Miyake, ACS Appl. Mater. Interfaces 2012, 4, 3325.

[8] A. Facchetti, Materials Today 2013, 16, 123.

[9] See definition of CTS under: http://goldbook.iupac.org/C01006.html

[10] K. Vandewal, A. Gadisa, W. D. Oosterbaan, S. Bertho, F. Banishoeib, I. Van Severen, L. Lutsen, T. J. Cleij, D. Vanderzande, J. V. Manca, Advanced Functional Materials 2008, 18, 2064.

[11] C. Yin, T. Kietzke, D. Neher, H. H. Horhold, Appl. Phys. Lett. 2007, 90, 092117.

[12] C. Yin, M. Schubert, S. Bange, B. Stiller, M. Castellani, D. Neher, M. Kumke, H. H. Horhold, J. Phys. Chem. C 2008, 112, 14607.

[13] J. M. Hodgkiss, A. R. Campbell, R. A. Marsh, A. Rao, S. Albert-Seifried, R. H. Friend, Phys. Rev. Lett. 2010, 104, 177701.

[14] S. Westenhoff, I. A. Howard, J. M. Hodgkiss, K. R. Kirov, H. A. Bronstein, C. K. Williams, N. C. Greenham, R. H. Friend, J. Am. Chem. Soc. 2008, 130, 13653.

[15] C. Deibel, T. Strobel, V. Dyakonov, Phys. Rev. Lett. 2009, 103, 036402.

[16] D. H. K. Murthy, M. Gao, M. J. W. Vermeulen, L. D. A. Siebbeles, T. J. Savenije, The Journal of Physical Chemistry C 2012, 116, 9214.

[17] A. A. Bakulin, A. Rao, V. G. Pavelyev, P. H. M. van Loosdrecht, M. S. Pshenichnikov, D. Niedzialek, J. r. m. Cornil, D. Beljonne, R. H. Friend, Science 2012, 335, 1340.

[18] M. C. Heiber, A. Dhinojwala, The Journal of Chemical Physics 2012, 137, 014903.

[19] H. Tamura, I. Burghardt, J. Am. Chem. Soc. 2013, 135, 16364.

[20] P. H. Wobkenberg, D. D. C. Bradley, D. Kronholm, J. C. Hummelen, D. M. de Leeuw, M. Colle, T. D. Anthopoulos, Synth. Met. 2008, 158, 468. 
[21] C.-Z. Li, C.-C. Chueh, H.-L. Yip, J. Zou, W.-C. Chen, A. K. Y. Jen, Journal of Materials Chemistry 2012, 22, 14976.

[22] C. R. McNeill, A. Abrusci, J. Zaumseil, R. Wilson, M. J. McKiernan, J. H. Burroughes, J. J. M. Halls, N. C. Greenham, R. H. Friend, Appl. Phys. Lett. 2007, 90, 193506.

[23] R. A. Marsh, C. Groves, N. C. Greenham, Journal of Applied Physics 2007, 101, 083509.

[24] H. Yan, Z. H. Chen, Y. Zheng, C. Newman, J. R. Quinn, F. Dotz, M. Kastler, A. Facchetti, Nature 2009, 457, 679.

[25] X. Guo, F. S. Kim, M. J. Seger, S. A. Jenekhe, M. D. Watson, Chemistry of Materials 2012, 24, 1434.

[26] S. Cho, J. Lee, M. Tong, J. H. Seo, C. Yang, Advanced Functional Materials 2011, 21, 1910.

[27] J. Li, Y. Zhao, H. S. Tan, Y. Guo, C.-A. Di, G. Yu, Y. Liu, M. Lin, S. H. Lim, Y. Zhou, H. Su, B. S. Ong, Sci. Rep. 2012, 2.

[28] J. H. Park, E. H. Jung, J. W. Jung, W. H. Jo, Advanced Materials 2013, 25, 2583.

[29] J. Lee, A. R. Han, H. Yu, T. J. Shin, C. Yang, J. H. Oh, J. Am. Chem. Soc. 2013, 135, 9540.

[30] R. Steyrleuthner, M. Schubert, F. Jaiser, J. C. Blakesley, Z. Chen, A. Facchetti, D. Neher, Advanced Materials 2010, 22, 2799.

[31] J. C. Blakesley, M. Schubert, R. Steyrleuthner, Z. H. Chen, A. Facchetti, D. Neher, Appl. Phys. Lett. 2011, 99, 183310.

[32] T. Schuettfort, S. Huettner, S. Lilliu, J. E. Macdonald, L. Thomsen, C. R. McNeill, Macromolecules 2011, 44, 1530.

[33] J. Rivnay, M. F. Toney, Y. Zheng, I. V. Kauvar, Z. H. Chen, V. Wagner, A. Facchetti, A. Salleo, Advanced Materials 2010, 22, 4359.

[34] J. Rivnay, R. Steyrleuthner, L. H. Jimison, A. Casadei, Z. H. Chen, M. F. Toney, A. Facchetti, D. Neher, A. Salleo, Macromolecules 2011, 44, 5246.

[35] R. Steyrleuthner, M. Schubert, I. Howard, B. Klaumünzer, K. Schilling, Z. Chen, P. Saalfrank, F. Laquai, A. Facchetti, D. Neher, J. Am. Chem. Soc. 2012, 134, 18303.

[36] J. B. Kim, S. Lee, M. F. Toney, Z. Chen, A. Facchetti, Y. S. Kim, Y.-L. Loo, Chemistry of Materials 2010, 22, 4931.

[37] S. Fabiano, Z. Chen, S. Vahedi, A. Facchetti, B. Pignataro, M. A. Loi, Journal of Materials Chemistry 2011, 21, 5891.

[38] J. R. Moore, S. Albert-Seifried, A. Rao, S. Massip, B. Watts, D. J. Morgan, R. H. Friend, C. R. McNeill, H. Sirringhaus, Advanced Energy Materials 2011, 1, 230.

[39] T. W. Holcombe, J. E. Norton, J. Rivnay, C. H. Woo, L. Goris, C. Piliego, G. Griffini, A. Sellinger, J.-L. Brédas, A. Salleo, J. M. J. Fréchet, J. Am. Chem. Soc. 2011, 133, 12106.

[40] M. Schubert, D. Dolfen, J. Frisch, S. Roland, R. Steyrleuthner, B. Stiller, Z. H. Chen, U. Scherf, N. Koch, A. Facchetti, D. Neher, Advanced Energy Materials 2012, 2, 369.

[41] K. Vandewal, S. Albrecht, E. T. Hoke, K. R. Graham, J. Widmer, J. D. Douglas, M. Schubert, W. R. Mateker, J. T. Bloking, G. F. Burkhard, A. Sellinger, J. M. J. Fréchet, A. Amassian, M. K. Riede, M. D. McGehee, D. Neher, A. Salleo, Nat. Mater. 2014, 13, 63.

[42] J. Lee, K. Vandewal, S. R. Yost, M. E. Bahlke, L. Goris, M. A. Baldo, J. V. Manca, T. Van Voorhis, J. Am. Chem. Soc. 2010, 132, 11878.

[43] L. Goris, K. Haenen, M. Nesladek, P. Wagner, D. Vanderzande, L. De Schepper, J. D'Haen, L. Lutsen, J. V. Manca, J. Mater. Sci. 2005, 40, 1413.

[44] J. J. Benson-Smith, L. Goris, K. Vandewal, K. Haenen, J. V. Manca, D. Vanderzande, D. D. C. Bradley, J. Nelson, Advanced Functional Materials 2007, 17, 451.

[45] K. Vandewal, K. Tvingstedt, A. Gadisa, O. Inganas, J. V. Manca, Nat. Mater. 2009, 8, 904. 
[46] B. A. Collins, J. E. Cochran, H. Yan, E. Gann, C. Hub, R. Fink, C. Wang, T.

Schuettfort, C. R. McNeill, M. L. Chabinyc, H. Ade, Nat. Mater. 2012, 11, 536.

[47] H. P. Yan, B. A. Collins, E. Gann, C. Wang, H. Ade, C. R. McNeill, ACS Nano 2012, $6,677$.

[48] D. P. McMahon, D. L. Cheung, A. Troisi, The Journal of Physical Chemistry Letters 2011, 2, 2737.

[49] F. C. Jamieson, E. B. Domingo, T. McCarthy-Ward, M. Heeney, N. Stingelin, J. R. Durrant, Chemical Science 2012, 3, 485.

[50] H. Sirringhaus, P. J. Brown, R. H. Friend, M. M. Nielsen, K. Bechgaard, B. M. W. Langeveld-Voss, A. J. H. Spiering, R. A. J. Janssen, E. W. Meijer, P. Herwig, D. M. de Leeuw, Nature 1999, 401, 685.

[51] J. L. Baker, L. H. Jimison, S. Mannsfeld, S. Volkman, S. Yin, V. Subramanian, A. Salleo, A. P. Alivisatos, M. F. Toney, Langmuir 2010, 26, 9146.

[52] M. R. Hammond, R. J. Kline, A. A. Herzing, L. J. Richter, D. S. Germack, H.-W. Ro, C. L. Soles, D. A. Fischer, T. Xu, L. Yu, M. F. Toney, D. M. DeLongchamp, ACS Nano 2012, 5,8248 .

[53] R. Steyrleuthner, R. Di Pietro, B. A. Collins, F. Polzer, S. Himmelberger, M. Schubert, Z. Chen, S. Zhang, A. Salleo, H. Ade, A. Facchetti, D. Neher, submitted.

[54] H. W. Ro, B. Akgun, B. T. O'Connor, M. Hammond, R. J. Kline, C. R. Snyder, S. K. Satija, A. L. Ayzner, M. F. Toney, C. L. Soles, D. M. DeLongchamp, Macromolecules 2012, $45,6587$.

[55] N. S. Sariciftci, L. Smilowitz, A. J. Heeger, F. Wudl, Science 1992, 258, 1474.

[56] R. Di Pietro, H. Sirringhaus, Advanced Materials 2012, 24, 3367.

[57] I. A. Howard, R. Mauer, M. Meister, F. Laquai, J. Am. Chem. Soc. 2010, 132, 14866.

[58] J. M. Guo, H. Ohkita, H. Benten, S. Ito, J. Am. Chem. Soc. 2010, 132, 6154.

[59] S. Fabiano, S. Himmelberger, M. Drees, Z. Chen, R. M. Altamimi, A. Salleo, M. A. Loi, A. Facchetti, Advanced Energy Materials 2014, DOI: 10.1002/aenm.201301409.

[60] B. P. Rand, D. Cheyns, K. Vasseur, N. C. Giebink, S. Mothy, Y. Yi, V. Coropceanu, D. Beljonne, J. Cornil, J.-L. Brédas, J. Genoe, Advanced Functional Materials 2012, 22, 2987.

[61] T. J. Savenije, J. E. Kroeze, X. N. Yang, J. Loos, Thin Solid Films 2006, 511, 2.

[62] F. Piersimoni, S. Chambon, K. Vandewal, R. Mens, T. Boonen, A. Gadisa, M.

Izquierdo, S. Filippone, B. Ruttens, J. D'Haen, N. Martin, L. Lutsen, D. Vanderzande, P. Adriaensens, J. V. Manca, J. Phys. Chem. C 2011, 115, 10873.

[63] G. D'Avino, S. Mothy, L. Muccioli, C. Zannoni, L. Wang, J. Cornil, D. Beljonne, F. Castet, The Journal of Physical Chemistry C 2013, 117, 12981.

[64] F. C. Spano, J. Chem. Phys. 2005, 122, 234701.

[65] S. T. Turner, P. Pingel, R. Steyrleuthner, E. J. W. Crossland, S. Ludwigs, D. Neher, Advanced Functional Materials 2011, 21, 4640.

[66] D. Caruso, A. Troisi, Proc. Natl. Acad. Sci. U. S. A. 2012, 109, 13498.

[67] H. Tamura, M. Tsukada, Physical Review B 2012, 85, 054301.

[68] H. Tamura, I. Burghardt, The Journal of Physical Chemistry C 2013, 117, 15020.

[69] R. F. Fink, J. Seibt, V. Engel, M. Renz, M. Kaupp, S. Lochbrunner, H. M. Zhao, J.

Pfister, F. Wurthner, B. Engels, J. Am. Chem. Soc. 2008, 130, 12858.

[70] D. Mori, H. Benten, I. Okada, H. Ohkita, S. Ito, Advanced Energy Materials 2014, DOI: 10.1002/aenm.201301006.

[71] https://github.com/EFTEMj

[72] P. Thevenaz, U. E. Ruttimann, M. Unser, Image Processing, IEEE Transactions on 1998, 7, 27.

[73] http://bigwww.epfl.ch/thevenaz/turboreg 
[74] E. Gann, A. T. Young, B. A. Collins, H. Yan, J. Nasiatka, H. A. Padmore, H. Ade, A. Hexemer, C. Wang, Review of Scientific Instruments 2012, 83, 045110

[75] A. L. D. Kilcoyne, T. Tyliszczak, W. F. Steele, S. Fakra, P. Hitchcock, K. Franck, E. Anderson, B. Harteneck, E. G. Rightor, G. E. Mitchell, A. P. Hitchcock, L. Yang, T. Warwick, H. Ade, Journal of Synchrotron Radiation 2003, 10, 125.

[76] B. A. Collins, Z. Li, J. R. Tumbleston, E. Gann, C. R. McNeill, H. Ade, Advanced Energy Materials 2013, 3, 65.

[77] A. Hexemer, W. Bras, J. Glossinger, E. Schaible, E. Gann, R. Kirian, A. MacDowell, M. Church, B. Rude, H. Padmore, Journal of Physics: Conference Series 2010, 247, 012007. 
Splitting excitons into free charges can be very efficient at polymer/fullerene interfaces but it becomes a challenging task when the fullerene is replaced by a polymeric acceptor. Here, we investigate the structural origin of the photocurrent generation in all-polymer solar cells consisting of P3HT and P(NDI2OD-T2). We find that a proper orientation of the polymer crystallites is needed to enable exciton dissociation and identify the geminate recombination of electron hole pairs and the incomplete quenching of P(NDI2OD-T2) excitons as major loss channels.

Keywords: photocurrent generation, charge-transfer state recombination, polymer crystals, alternative acceptors, organic solar cells

Marcel Schubert, Brian A. Collins, Hannah Mangold, Ian A. Howard, Wolfram Schindler, Koen Vandewal, Steffen Roland, Jan Behrends, Felix Kraffert, Robert Steyrleuthner, Zhihua Chen, Konstantinos Fostiropoulos, Robert Bittl, Alberto Salleo, Antonio Facchetti, Frédéric Laquai, Harald Ade, and Dieter Neher*

\section{Correlated Donor/Acceptor Crystal Orientation Controls Photocurrent Generation in All-Polymer Solar Cells}

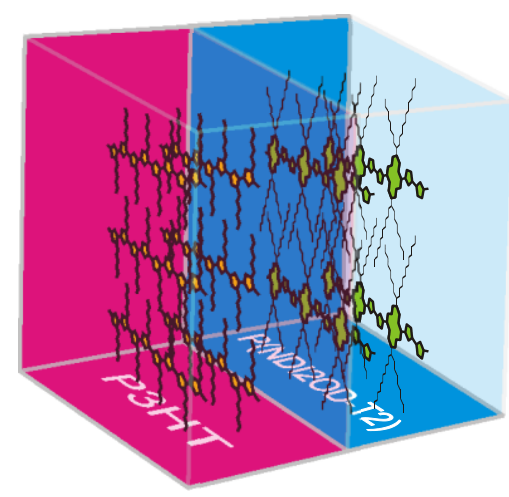

